\title{
Violences stratégiques et violences désorganisées dans la région de Urabá en Colombie
}

Gérard Martin

\section{(2) OpenEdition \\ 1 Journals}

Édition électronique

URL : http://journals.openedition.org/conflits/2166

DOI : 10.4000/conflits.2166

ISSN : $1777-5345$

Éditeur :

CCLS - Centre d'études sur les conflits lilberté et sécurité, L'Harmattan

Édition imprimée

Date de publication : 15 mai 1997

ISSN : 1157-996X

Référence électronique

Gérard Martin, «Violences stratégiques et violences désorganisées dans la région de Urabá en Colombie », Cultures \& Conflits [En ligne], 24-25 | hiver 1996- printemps 1997, mis en ligne le 27 mars 2007, consulté le 30 mars 2021. URL : http://journals.openedition.org/conflits/2166 ; DOI : https:// doi.org/10.4000/conflits.2166

Ce document a été généré automatiquement le 30 mars 2021.

Creative Commons License 


\title{
Violences stratégiques et violences désorganisées dans la région de Urabá en Colombie
}

\author{
Gérard Martin
}

1 Depuis le milieu des années quatre-vingt, la Colombie vit au rythme de 25.000 à 30.000 morts violents par an ${ }^{1}$. Avec un taux d'homicide de 70 pour 100.000, la Colombie est, depuis dix ans, un des pays les plus violents au monde, exception faite des pays en guerre déclarée ou en guerre civile ouverte. Durant la période 1985-1996, le nombre de victimes s'élève à 250.000 , toutes violences confondues (contre quelque 85.000 pour la décennie précédente). La gravité de la situation n'est pas sans rappeler la période de semi-guerre civile des années 1947-1963, plus connue sous le nom de La Violencia et qui a fait 200.000 morts $^{2}$. Toutefois, les événements de La Violencia affichaient au moins un semblant d'unité et s'inscrivaient dans une confrontation politique entre libéraux et conservateurs, tandis que la crise actuelle se caractérise par une telle multiplicité de protagonistes, d'enjeux et des lieux de la violence, qu'une image globale n'arrive pas à s'imposer.

2 La dépolitisation de la violence est particulièrement forte et les interférences entre des protagonistes supposés politiques (guérilla, paramilitaires) et non-politiques (narcotrafiquants, délinquance commune) sont permanentes ${ }^{3}$. Seules $15 \%$ des victimes relèvent de confrontations entre guérilla et force publique. Il ne s'agit donc pas d'une guerre civile. Ainsi, la crise que traverse la Colombie est plus difficile à analyser en termes politiques que celles qu'ont connues le Guatemala, le Salvador ou le Nicaragua. Par ailleurs, malgré la généralisation de la violence, la Colombie ne se désintègre pas et continue à mettre en avant et à revendiquer son statut d'Etat de droit et l'existence de régulations démocratiques. La situation se différencie également de celle de pays comme la Somalie, le Liberia et la Sierra Leone, où des bandes armées agissent dans " un vide " après l'effondrement quasi total des structures étatiques.

3 La spécificité de la crise colombienne a été décrite par Daniel Pécaut comme une situation de violence généralisée ${ }^{4}$, se traduisant dans maintes zones rurales et urbaines 
par des situations de terreur ${ }^{5}$. Les frontières entre luttes armées, violences sociales, criminalité organisée et délinquance commune ont disparu. Des groupes armés bien structurés comme des organisations paramilitaires, d'autodéfense et de guérilla se livrent des guerres locales pour imposer ou consolider leur contrôle sur des ressources et des possessions diverses ${ }^{6}$. S'y mêlent toute une série d'acteurs plus désorganisés, dont les motifs peuvent être des plus banals jusqu'aux plus politiques. Violences politiques et non-politiques, organisées et inorganisées, entrent en résonance et finissent par redéfinir le contexte dans lequel la société se reproduit ${ }^{7}$. La violence apparaît désormais non plus comme un phénomène qu'on peut ramener à des causes précises, mais comme formant le contexte même dans lequel la société évolue.

4 Cette description se distingue de deux approches différentes. L'une tente de réinterpréter la violence actuelle, au regard d'un enjeu central ou d'un conflit dominant $^{8}$. Or, la multiplicité des acteurs de la violence et la complexité même de la crise est telle qu'il s'avère illusoire d'expliquer la violence par des problèmes de pauvreté, ou encore de la concevoir en terme de conflit de classe ou de confrontation politique. Des conflits sociaux se mêlent certes à la violence, mais celle-ci est le plus souvent parasitaire de ces conflits et ne se situe pas dans leur prolongement, comme c'est en général le cas dans des situations de violence collective ${ }^{9}$. L'autre approche, culturaliste, présente la société colombienne comme une société historiquement dominée par la violence. En Colombie, il s'agit certes d'une situation où la violence s'impose à un tel degré que se crée l'image, au moins parmi ceux que la subissent, d'une société dominée par " la violence ", comme un tout irréductible et incontrôlable. Mais il ne s'agit pas moins d'une situation hautement stratégique, où la violence est mise en œuvre par des protagonistes qui interviennent sur des théâtres précis en fonction d'enjeux particuliers. Leurs choix et stratégies sont analysables, bien que des visées et des actions strictement politiques y soient difficiles à isoler ${ }^{10}$.

5 Une dimension centrale de la violence actuelle, en partie responsable de son escalade rapide et de son intensité, est la manière dont la violence organisée entretient en permanence des liens circulaires avec des formes de violence plus désorganisée et, de façon plus générale, avec des phénomènes de désorganisation sociale. Ce qui nous intéresse ici, c'est de savoir dans quelle mesure la multiplication des phénomènes de violence désorganisée a été une conséquence de la percée spectaculaire des acteurs armés, bien que les deux dimensions n'aient jamais cessé de se renforcer mutuellement. Selon Daniel Pécaut, " (...) la violence mise en œuvre par les protagonistes organisés constitue le cadre dans lequel se développe la violence désorganisée (qui) contribue à élargir le champ de la violence organisée. L'une et l'autre se renforcent mutuellement $" 11$.

6 Nous nous proposons d'étudier ici à la fois la dimension stratégique de la violence et les liens circulaires qui se nouent en permanence entre violence stratégique, violence anomique et formes de désorganisation sociale ${ }^{12}$. Nous nous concentrerons sur les phénomènes de violence qui se sont succédés depuis bientôt vingt ans dans la région d'Urabá, zone particulièrement affectée par la crise actuelle. Après quelques remarques sur l'évolution récente de la région en tant que zone de colonisation récente, de guérillas et de narcotrafic, nous distinguerons, sur le plan de la violence organisée, cinq épisodes consécutifs de guerres locales : guerre géostratégique, guerre syndicale, première et deuxième guerre anti-subversive, guerre de réinsertion. Nous montrerons que ces guerres locales opposent, dans des configurations changeantes, des acteurs 
différents pour imposer des contrôles territoriaux et d'autres formes d'hégémonie locale. Nous analyserons ensuite brièvement le rôle de la force publique. Nous décrirons enfin quelques formes de violence déstructurée et les rapports existants entre celles-ci et la violence plus organisée.

La région d'Urabá

7 La violence touche désormais presque toutes les régions de la Colombie. Elle s'exprime de manière particulièrement forte dans certains lieux déterminés, notamment dans les villes $^{13}$ et dans les zones de colonisation récente, comme la région d'Urabá, le bajo Cauca, le Magdalena medio. Les villes comme les régions de colonisation récente, connaissent, depuis les années cinquante, des croissances démographiques très rapides s'accompagnant de phénomènes complexes de formation de larges couches populaires ${ }^{14}$ et de désorganisation sociale ${ }^{15}$. Ces nouvelles sociétés urbaines et rurales se construisent dans la précarité autour de migrants d'origines régionales souvent très hétérogènes ; d'où un déficit de cohésion sociale d'autant plus important que ces phénomènes évoluent dans un double contexte : d'une part, celui d'un processus de modernisation négative, où urbanisation et sécularisation extrêmement rapides ne s'accompagnent guère de nouvelles formes d'encadrement institutionnel et, d'autre part, celui d'une faiblesse historique de l'Etat, marqué par le clientélisme et la corruption et dont l'extrême inefficacité de l'administration judiciaire a toujours été un des aspects les plus préoccupants. Ainsi, les grandes villes et les zones de colonisation récente sont particulièrement sensibles aux phénomènes de délinquance, et de violence en général, et à l'implantation de nouveaux protagonistes armés, notamment la guérilla et les réseaux mafieux.

Une zone de colonisation récente

Région périphérique, située au nord-ouest de la Colombie, sur la frontière avec le Panama, la zone d'Urabá s'étend autour d'un golfe, qui forme un magnifique port naturel sur la mer Caraïbe ${ }^{16}$. Cette région présente un intérêt géostratégique considérable, puisqu'elle est proche du canal de Panama et que l'on évoque périodiquement la possibilité d'y percer un deuxième canal transocéanique. De plus, la route transaméricaine, la pan-american highway, qui doit relier les deux parties du continent américain, doit passer par Urabá. Le destin de la région est donc bien de devenir un carrefour routier et un lieu de transit de marchandises et de populations. Pour le moment, la zone frontalière colombo-panaméenne est encore largement couverte de forêt tropicale. Guérilla, narcotrafiquants, contrebandiers, marines américaines et réseaux d'immigration clandestine s'y côtoient.

Urabá a été, jusque dans les années cinquante, une région peu peuplée, marginalement intégrée à l'économie nationale. L'ouverture massive à la colonisation ne date que de 1960. La région se transforme alors, du jour au lendemain, en une très importante zone de migration interne, suite à l'installation de 300 plantations de bananes par des investisseurs essentiellement colombiens, qui profitent d'un important programme de crédits et d'assistance technique de la United Fruit Brands. Le secteur bananier devient aussitôt le moteur de l'économie régionale et un des plus grands pôles agro-industriels du pays avec quelque 13.000 travailleurs directs et 7.000 travailleurs indirects ${ }^{17}$.

Certains citadins, notamment de Medellín (chef-lieu du département d'Antioquia dont la région d'Urabá fait partie), disposaient déjà avant 1960 de grands domaines d'élevage dans la région. Mais c'est avec l'agro-industrie bananière qu'Urabá devient une région très convoitée par des personnes à la recherche d'occasions d'investissement. Parmi la 
première génération de planteurs, on trouve, outre des investisseurs plus occasionnels et des aventuriers, des membres de plusieurs grandes familles politiques et économiques du pays. Si l'on assiste à la constitution d'une élite régionale, il s'agit toutefois d'une élite essentiellement absentéiste, habitant Medellín ou d'autres grandes villes, gérant leurs affaires dans la zone d'Urabá par l'intermédiaire d'administrateurs et de maisons de commercialisation à $100 \%$ colombiennes, qui parviennent rapidement à déplacer la United Fruit Brands de la région.

11 Des dizaines de milliers de migrants s'installent à partir 1960 dans la région, attirés par la demande de main d'œuvre salariée dans les bananeraies. Les travailleurs sont en grande partie hébergés dans des baraquements précaires installés à l'intérieur des plantations. D'autres migrants s'aventurent sur les fronts de colonisation qui s'ouvrent en parallèle un peu partout dans la région. Des villes champignons naissent autour de l'agro-industrie (Turbo, Apartado, Chigorodo), et abritent actuellement près de la moitié de la population régionale, qui s'élève à quelque 400.000 habitants ${ }^{18}$.

12 La société régionale se construit dans la rapidité et le désordre, ce qui ne va pas sans conflit. La distribution des ressources, en particulier des terres rurales et urbaines, se fait sur la base d'initiatives privées, faiblement encadrées par les régulations institutionnelles. En résultent d'innombrables litiges entre migrants et spéculateurs fonciers, mais aussi au sein même des migrants, et avec les populations indigènes. Les relations de travail et les conditions de vie dans les baraquements des bananeraies sont en même temps porteuses de forts conflits sociaux, par ailleurs peu structurés. Enfin, face à ces vagues de migrants, les infrastructures et les services publics resteront longtemps extrêmement précaires, tandis que les institutions, notamment judiciaires, manqueront de ressources humaines et logistiques pour faire face aux nouvelles demandes. Toutefois, le statut instable et le caractère hétérogène des migrants aidant, les conflits sociaux ne s'exprimeront guère, au long les années soixante et soixante-dix, de manière collective ${ }^{19}$.

Périphérique et très isolée de son hinterland, cette nouvelle société n'assiste pas moins à la réinventions rapide de l'ancien pays politique. Des barons électoraux du Parti Libéral - parti qui domine traditionnellement le paysage politique du nord-ouest de la Colombie - vont utiliser Urabá comme une réserve électorale. Tirant leur force de Medellín, ils transforment les emplois de fonctionnaires, les syndicats et les associations, en autant de relais politiques dirigés par des petits middle-men locaux. Ces réseaux de clientélisme politique permettront les premières formes d'intégration verticale entre le centre et la périphérie. Toutefois, dans un contexte de faible cohésion sociale et d'absentéisme chronique des élites économiques et politiques régionales, la faiblesse des formes d'encadrement restera de rigueur.

Une région de violence chronique

Durant les années soixante et soixante-dix, l'Etat n'a jamais entamé une politique de sécurité interne sous la forme d'un renforcement et d'une modernisation de l'administration judiciaire et de la force publique. Sur le plan de la gestion institutionnelle des problèmes de violence, rien d'essentiel n'est venu marquer, sous le régime du Front National (1958-1974), la fin de La Violencia ${ }^{20}$. Les stratégies introduites pour combattre la criminalité et la délinquance commune restent extrêmement aléatoires et rudimentaires. Aux campagnes de " nettoyage " du bandolerisme (1957-1963), du banditisme et autres " antisociaux ", s'ajoutent des logiques " d'éradication " de la guérilla et de " répression " de la délinquance commune. 
L'impunité règne et la violence gardera, sur le plan national, toujours un taux relativement élevé.

15 A Urabá, durant les années soixante et soixante-dix, la violence présente un caractère chronique. Dans le contexte du boom et du débordement populaire, les processus légaux ne s'imposent que très partiellement face à la violence privée. Devant l'inefficacité de l'administration de la justice, on a recours à la justice privée. Ce recours s'inscrit dans un " savoir-faire " qui veut que les disputes et les différents soient résolus sur un plan personnel. Dans la mesure où l'ouverture rapide à la colonisation est vécue en termes de prédation, l'intervention de la justice dans les conflits et des litiges est perçue comme une atteinte au droit individuel. Apparaissent aussi, par période, des escadrons de la mort, qui agissent notamment à l'encontre de la délinquance commune, à l'initiative sans doute de certains secteurs de la société régionale. Cependant, les violences gardaient en général un caractère " modéré ", et elles étaient perçues comme relevant de conflits limités et définis sur le plan personnel, social, politique ou économique, ou relevant de phénomènes de désorganisation sociale classique propre à une zone de frontière (beuveries, bagarres, défis).

La situation de violence chronique des années soixante et soixante-dix a certes facilité le passage, dans les années quatre-vingt, à une conjoncture de violence généralisée, mais elle ne suffit pas pour autant à l'expliquer ${ }^{21}$. Il s'agit là, en effet, d'un véritable changement de Gestallt, qui se traduit tant sur le plan de l'intensité que sur le plan des expressions de la violence. A Urabá, dans la zone bananière, le taux annuel d'homicide fait plus que tripler : il passe de 50 à 100 pour 100.000 , au cours des années soixante et soixante-dix, de 300 à 450 pour 100.000, pour les années 1985-9522. Mais au-delà de la multiplication des violences, la nouvelle situation s'exprime par l'apparition d'une dimension très importante de la violence organisée, notamment autour de l'économie de la drogue et des groupes de guérilla actifs dans la région.

Une zone d'implantation de la guérilla

Urabá a connu, depuis les années quarante, un problème chronique de groupes de guérilla et de formes de banditisme. Les zones de haute montagne, de marais et de forêts denses qui entourent les plaines fertiles d'Urabá rendent la communication avec l'intérieur du pays extrêmement difficile. Ils se prêtent, par contre, particulièrement bien aux activités de bandes d'irréguliers de tous ordres. Il convient brièvement d'en distinguer les phases successives, pour mieux saisir la spécificité de la guérilla actuelle.

Durant la guerre civile des années quarante et cinquante, une poignée de groupes de guérilla d'inspiration libérale (la chusma), financés et soutenus par des dirigeants régionaux libéraux opposés au régime conservateur, s'implantent dans la région, encore peu habitée. Ces groupes, qui comptent entre 20 à 50 membres, agissent selon une logique de guerre civile. Ils mènent leur lutte davantage sous forme d'actions et de massacres contre des conservateurs, que sous forme de combats directs contre la police ou contre les bandes armées conservatrices (contra-chusma). Ces derniers, à leur tour, se livrent à des actions et des massacres contre des libéraux.

Après l'amnistie et l'abandon des armes sous le régime de Rojas Pinilla (1953-1957), certains irréductibles (libéraux ou conservateurs) se maintiennent comme une sorte de corps-francs dans les parties limitrophes de la région. Une guerre sale et sans merci (1957-1963) est livrée contre ces bandoleros, où participent parfois leurs anciens patrons politiques, qui voient dans leur insoumission un défi au rétablissement de leur pouvoir et de leur respectabilité. 
20 Au début des années soixante, plusieurs " foyers " (focos) de guérilla communiste, inspirés par les révolutions chinoise et cubaine, s'implantent dans la région. La majorité de ces groupuscules échouent en raison de leur mauvaise organisation, de conflits internes, d'un manque chronique d'hommes, d'armes et d'argent, mais aussi du fait de la répression par la force publique et de l'hostilité de la population locale, qui fait les frais de l'aventure révolutionnaire en devenant la première victime des opérations de répression souvent brutales.

21 Fin des années soixante, début des années soixante-dix, deux nouveaux groupes de guérilla parviennent à s'installer durablement dans certaines parties de la région. Il s'agit, d'une part, de la guérilla communiste des FARC, bras armé du traditionnel Parti Communiste (PCC) et, d'autre part, de la guérilla maoïste de l'EPL, bras armé du Parti Communiste marxiste-léniniste (PC-ml) qui se veut clandestin et dont les principaux dirigeants avaient été expulsés du PCC.

Quand l'Etat Major des FARC décide de créer, dans le sud d'Urabá, son 5ème Front ${ }^{22}$, le Parti Communiste y dispose déjà de quelques conseillers municipaux ${ }^{23}$, de diverses associations de quartiers et d'un petit syndicat de travailleurs bananiers (Sintrabanano), dont certains membres étaient des relais de confiance pour les FARC. L'EPL ayant sa base principale dans les montagnes du département limitrophe de Cordoba, réalise des incursions vers le nord d'Urabá. Mais les maoïstes, qui prônent par ailleurs l'abstention électorale, ne contrôlent pas encore d'organisations de base dans la région, où ils ne disposent que d'une poignée de dirigeants politiques clandestins.

Jusqu'à la fin des années soixante-dix, FARC et EPL ont entre 20 et 40 combattants dans la région. Leur arsenal est peu important. A l'exception de certaines aides extérieures, ces groupes n'ont alors d'autres ressources que celles provenant des prises d'assaut des caisses du Crédit rural (Caja Agraria) ou des contrats avec des éleveurs, dont ils protègent les domaines contre le vol de bétail ou contre l'invasion par les colons. Les contrôles territoriaux qu'exercent ces groupes restent périphériques. Leur capacité d'encadrement de la population est peu importante et concerne essentiellement des colons ou des paysans, mais pas des secteurs salariés. Leur capacité de nuisance n'en est pas moins considérable et provoque, par périodes, des campagnes militaires, des couvre-feux ou l'installation de maires militaires dans les villes-champignons.

24 A Urabá dans les années quatre-vingt, à l'instar de ce que l'on constate dans le reste du pays, les FARC et l'EPL vont consciemment abandonner leur marginalité. Ils visent maintenant à établir des contrôles sur les secteurs modernes de l'économie régionale, en particulier sur l'agro-industrie bananière. Les attaques peu rentables des agences du Crédit rural sont remplacées par le racket et les prises d'otage à l'encontre du secteur bananier et des éleveurs, des commerçants, des transporteurs, etc. La guérilla vise aussi les ressources procurées par l'économie de la drogue (voir ci-dessous). Le recours à ces diverses pratiques, nouvelles et hautement rentables, explique en partie les avancées territoriales de la guérilla, la modernisation spectaculaire de son arsenal et l'importance croissance des effectifs de combattants. A la fin des années quatre-vingt, les FARC et l'EPL disposent chacun de divers fronts et de plusieurs centaines de combattants bien armés dans la région.

Cette transformation de la guérilla s'accompagne en même temps d'une forme militaire plus offensive ${ }^{24}$. Elle combat la force publique dans les zones rurales (elle obtient le démantèlement des postes de police rurale pour raison d'insécurité) et s'implante, sous forme de milices, dans les quartiers populaires des villes-champignons de la région. Elle 
entend désormais contrôler militairement des territoires et des populations, tout en se construisant une nouvelle légitimité politique par le chantage à la terreur à l'encontre des populations habitant ces zones.

Ainsi, le financement, la structure de l'organisation, le caractère des forces combattantes, la relation avec la population civile, les relations avec les autres acteurs armés se transforment profondément à partir du début des années quatre-vingt. L'enhardissement et l'élargissement de la guérilla va ouvrir l'espace à de nombreux conflits nouveaux, dont certains prennent le caractère de véritables guerres locales. A Urabá, les FARC et l'EPL restent par ailleurs à tel point divisés qu'ils ne cessent de s'affronter militairement entre eux par populations interposées ${ }^{25}$. Dans la région d'Urabá, cette guerre entre FARC et EPL ajoute une dimension qui complique singulièrement le panorama de la violence.

Une zone de narcotrafic

La région d'Urabá a connu, par sa position géostratégique, une longue histoire de contrebande. Le port de Turbo devient après la deuxième guerre mondiale un pittoresque centre de contrebande de tissus, de cigarettes, de whisky, d'appareils électroménagers, de pièces détachées de voitures et d'autres produits de luxe en provenance de la zone du canal de Panama, des Antilles et des Etats-Unis. Durant les années soixante, des réseaux délinquants opérant à partir de Medellín parviennent à contrôler les affaires.

Ces mêmes réseaux participent ensuite à la transformation d'Urabá, en plaque tournante pour l'économie de la drogue ${ }^{26}$. Au début des années soixante-dix, Urabá s'affiche, comme d'autres parties de la côte atlantique colombienne, comme une importante zone de production et d'exportation de marihuana vers les Etats-Unis. Des plantations de coca et des laboratoires de cocaïne fonctionnent, durant toutes les années quatre-vingt, sur la rive gauche du golfe d'Urabá ${ }^{27}$. La région devient en effet, de par sa position géostratégique, très convoitée par les narcotrafiquants. Ceux-ci achètent, souvent par des intermédiaires, des domaines d'élevage et même des bananeraies. Il s'agit, par delà la volonté de blanchir des capitaux, d'établir des bases dans la région, comme l'ont fait Fidel Castaño, José Ocampo et Pablo Escobar ${ }^{28}$.

A Urabá aussi, l'économie de la drogue présente de multiples volets : production de coca, laboratoires de cocaïne, contrôle des transports, blanchiment de l'argent de la drogue, etc. Dans chacun de ces volets, interviennent plusieurs réseaux de narcotrafiquants. Chacun de ces réseaux met en place une configuration particulière (toujours instable, toujours changeante) d'alliances avec d'autres concurrents pour le pouvoir, d'infiltration de l'administration, de corruption des hommes politiques. Certains éleveurs, planteurs, commerçants, hommes politiques ou représentants de la force publique dans la région, deviennent, à partir des années soixante-dix, partie prenante des bonnes affaires du narcotrafic. Des paysans se lancent dans la culture de la drogue, des migrants travaillent dans les laboratoires, des travailleurs portuaires jouent un rôle dans le transport de la drogue pour le Panama ou ailleurs.

En ce qui concerne les rapports entre guérilla et réseaux mafieux, ils sont dans l'Urabá, comme dans le reste du pays, ambigus. Les narcotrafiquants ont par moment passé des accords strictement instrumentaux avec l'un ou l'autre groupe de guérilla (protection de laboratoires, trafic d'armes, transports de drogue). Or, la concurrence pour des territoires et les marchés illégaux finit toujours par provoquer des conflits, sinon des véritables guerres locales. Ce sont en partie aussi des provocations et des excès de la 
guérilla contre les intérêts de certains narcotrafiquants (vol de bétail, racket, enlèvement contre rançon ou assassinat de proches) qui provoquent des actions d'extermination contre les supposées bases sociales de la guérilla par des groupes paramilitaires. Ces groupes paramilitaires s'organisent selon des intérêts stratégiques locaux, souvent à l'initiative de narcotrafiquants, mais toujours en accord avec d'autres secteurs de la société régionale et selon des alliances circonstancielles avec des segments de la police ou de l'armée.

31 L'essor de l'économie de la drogue, véritable boite de Pandore, affecte tous les secteurs de la société. Il en résulte aussi que la relation entre économie de la drogue et phénomènes de violence n'est jamais simple.

Cinq guerres locales

32 A Urabá se présentent donc, à partir du début des années quatre-vingt, dans le contexte d'une société frontalière encore faiblement constituée et précairement encadrée par des régulations institutionnelles, des groupes armés privés, notamment la guérilla et des réseaux mafieux. Ceux-ci vont opérer pour imposer leur contrôle sur les ressources régionales légales (terres, populations, administrations municipales) ou illégales (trafic d'armes, narcotrafic, marchés de racket ou d'impôts révolutionnaires). Cette prolifération de bandes armées ne met pas seulement en échec le monopole de l'Etat sur la violence, mais elle provoque un enchaînement continu de guerres locales et une situation de terreur permanente. A cela plusieurs raisons.

D'abord, puisqu'il y a plusieurs concurrents pour le pouvoir, les contrôles territoriaux que les groupes armés tentent d'imposer gardent toujours un caractère instable ; ceuxci doivent s'accorder sur des partages de territoires et de ressources, ce qui se réalise logiquement par le biais des armes. Ensuite, le contrôle territorial passe par l'instauration de formes d'emprises fortes sur la population locale qui s'imposent inévitablement par l'usage de la violence arbitraire, sinon de la terreur, qui prend périodiquement la forme de massacres, faisant jusqu'à 35 morts à la fois. Enfin, l'Etat est malgré tout présent à Urabá. La force publique s'arrange certes pour cohabiter sans trop de dégâts avec les nouveaux réseaux d'emprise, mais elle n'intervient pas moins militairement quand son monopole (déjà très partiel) est trop ouvertement mis en échec.

Le panorama de la violence organisée est extrêmement complexe et fluide, comme la succession de cinq guerres locales ayant lieu dans la région durant les vingt dernières années permet de l'illustrer. La situation ne cesse d'évoluer, et de prendre des formes nouvelles. On assiste notamment à des retournements d'alliances entre acteurs stratégiques, mais aussi entre ceux-ci et leurs supposées bases d'appui. Toutefois, au lieu de se représenter la situation par une image de flou et de désordre total, le rappel de ces guerres locales permet aussi de montrer la part de la violence stratégique dans la situation actuelle, en mettant en scène les protagonistes et leurs choix spécifiques.

Il s'agit ici nécessairement d'une présentation schématique puisque toute division en épisodes de guerre locale est relativement arbitraire. Les épisodes se prolongent, se juxtaposent et s'entremêlent d'une manière plus compliquée que nous ne pouvons l'indiquer ici. De plus, d'autres guerres locales se déroulent en même temps, dans d'autres parties de la région sans que nous les mentionnions, car nos propos se limitent à la zone bananière proprement dite.

Une guerre géostratégique (1978-80) 

lient à une fraction de l'EPL présente dans la région. Le conflit qui résulte de cette 'désertion' - commenté par les deux factions en des termes particulièrement haineux dans leurs journaux respectifs - apparaît à première vue comme un autre épisode des luttes idéologiques intra et inter-factionnelle de l'extrême gauche, notamment entre communistes et maoïstes, très courantes en Colombie dans les années soixante-dix ${ }^{29}$. Or, l'essentiel est ailleurs. Jusqu'alors, l'EPL et les FARC tenaient la région en fonction d'un partage en zones d'influences exclusives : le Sud et la zone bananière pour les FARC, le Nord et la Serrania del Abibe pour l'EPL, avec une frontière virtuelle à Turbo. La désertion remet cet équilibre en cause. L'EPL obtient, grâce aux déserteurs des FARC, une connaissance du terrain et des hommes de confiance de ce mouvement au sein de la population de la zone bananière, considérée par les FARC comme relevant de leur territoire. car situé sur la route de pénétration centrale, là où les collines de la Serrania del Abibe s'approchent au plus près du golfe d'Urabá. Le contrôle des plantations et des domaines qui constituent ce passage permet un accès rapide à la fois aux abords du golfe, pour participer au trafic d'armes et de drogues, ainsi qu'à la zone centrale, où se trouvent les bananeraies et les principales villes nouvelles de la région. Il s'agit donc essentiellement d'un conflit géostratégique entre EPL et FARC, bien qu'il n'y ait pas de doute que d'autres acteurs, en particulier ceux liés à l'essor de l'économie de la drogue, agissent parallèlement en coulisses ${ }^{30}$. dirigeants civiques et d'autres relais de l'une ou l'autre faction parmi la population, tous accusés de trahison, de délation etc. Ensuite, autour des enjeux géostratégiques proprement dits, les factions rivales visent aussi des administrateurs de bananeraies ou de domaines d'élevage, des inspecteurs départementaux de police, des travailleurs bananiers ou des habitants de certains hameaux sous l'influence de la faction rivale. Cet épisode fait plusieurs dizaines de morts.

La guerre syndicale (1985-1986)

39 La guerre syndicale relève de nouveau essentiellement d'un conflit territorial entre FARC et EPL, avant que les jeux stratégiques se complexifient singulièrement. Cela dit, elle se présente cette fois sous la forme de l'appropriation d'un espace social, celui du champ syndical des travailleurs bananiers. Les syndicats des travailleurs bananiers s'organisent par ailleurs en partie à travers cette même guerre.

40 La lutte pour le contrôle syndical des 12.000 salariés de l'agro-industrie bananière apparaît à première vue comme un conflit social classique, bien que polarisé à l'extrême ${ }^{31}$. Des dirigeants politiques de l'extrême gauche, de formation universitaire, arrivent à s'imposer (de manière fort peu démocratique) au début des années quatrevingt, à certaines organisations syndicales des travailleurs bananiers, jusque là encadrées par une génération de dirigeants qui n'étaient autres que les petits middlemen mis en place par les barons électoraux des partis politiques traditionnels. Ceci dans le double contexte d'une conjoncture florissante du secteur bananier et des pourparlers de paix entamés avec la guérilla par le gouvernement de Belisario Betancur (1982-1986).

41 Les nouveaux dirigeants, une fois les anciens leaders écartés et les syndicats cooptés, exigent, à partir 1984, des conventions collectives pour l'ensemble des 300 bananeraies, 
alors que jusqu'alors à peine $10 \%$ des plantations en bénéficiaient ${ }^{32}$. Le processus s'accompagne d'une syndicalisation massive des travailleurs et de mouvements de grèves paralysant par périodes tout le secteur et provoquant des tensions extrêmes avec les planteurs, dont la majorité avait jusque là systématiquement refusé que des syndicats s'installent sur leurs plantations. Avec l'intervention du Ministère de Travail, syndicats et patrons finissent par signer, en 1986, des conventions collectives couvrant la quasi-totalité du salariat bananier ${ }^{33}$.

Une interprétation institutionnelle, un conflit classique de rotation des dirigeants syndicaux et une lutte pour l'amélioration des conditions de travail, ne rend pas compte de la complexité des intérêts en jeu. La dimension bien réelle d'un conflit du travail est ainsi rapidement reléguée au second plan du fait d'un projet d'expansion politico-militaire qui oppose, par le biais du champ laboral, les deux factions d'extrême gauche, chacune cherchant à imposer, plantation par plantation, son propre syndicat ${ }^{34}$.

L'enjeu est de taille car il n'implique pas seulement le partage du contrôle financier des cotisations syndicales ${ }^{35}$, mais aussi celui d'une implantation territoriale stratégique au cœur même de la région d'Urabá. Cette implantation implique l'encadrement des 35.000 personnes, familles des travailleurs inclus, habitant désormais à l'intérieur des campements des quelque 300 plantations. Ce contrôle permet la construction de relais, de bases de recrutement, de lieu d'entraînement ou la présence clandestine de la guérilla à l'intérieur des bananeraies, ainsi qu'un contrôle électoral sur les travailleurs (ou, dans le cas des maoïstes, un contrôle abstentionniste). Ce 'clientélisme armé' permet un contrôle particulièrement efficace, car complètement décentralisé, par le biais de comités syndicaux installés dans chaque plantation.

A la confrontation entre les deux bandes pour le contrôle du champ syndical se mêlent très tôt des forces obscures, agissant pour le compte des intérêts bananiers, politiques ou autres. La situation se traduit en 1985-86 par un cycle infernal d'assassinats de dirigeants syndicaux, de membres de comités syndicaux, d'administrateurs, de contremaîtres et de travailleurs. Des massacres de 3 à 7 personnes sont perpétrés dans les bananeraies. Des attaques à la grenade ont lieu dans des bars à Currulao, coups de mains visant des travailleurs 'appartenant' à la faction rivale. Les sièges des syndicats sont attaqués, sans que personne n'arrive plus à comprendre par qui. La région apprend à vivre avec la terreur. Urabá s'impose comme une des régions les plus violentes du pays.

Une paix armée s'installe fin 1986 entre les deux factions, à l'occasion de la signature des conventions collectives, qui formalisent provisoirement le partage des territoires bananiers. Aux maoïstes revient alors, à travers leur syndicat Sintagro, 75\% des plantations ; aux communistes, à travers leur syndicat Sintrabanano, $25 \%$ des plantations. Ce partage inégal n'était acceptable pour les communistes que dans la mesure où ils obtenaient en même temps, par le biais de l'UP', lors des élections municipales de $1986^{37}$, un important début de contrôle sur les principales mairies de la région et pouvaient ainsi compenser leur pertes syndicales ${ }^{38}$.

La première guerre anti-subversive (1986-1989)

Tandis que la guerre géostratégique et la guerre syndicale étaient essentiellement des conflits à deux parties, la première guerre anti-subversive réunit, dès le début, au moins quatre parties : FARC, EPL, réseaux de narcotrafiquants et élites régionales. La situation se complique alors considérablement ${ }^{39}$. Les enjeux deviennent maintenant si multiples, les alliances entre les protagonistes si changeantes, les interférences entre 
stratégies et leurs effets pervers si complexes, que la violence (organisée) à Urabá n'a probablement jamais été aussi peu déchiffrable que durant les années 1986-89.

Les élites politiques et économiques régionales avaient eu à souffrir de pertes importantes en 1984-86 lors de la guerre syndicale : l'extrême gauche était arrivée à imposer des formes d'emprise forte sur le salariat agro-industriel, à déplacer d'autres réseaux de clientèle traditionnels, à menacer le contrôle libéral sur l'administration des villes nouvelles. Les grèves avaient provoqué des pertes considérables, certains petits planteurs faisant même faillite. A cela, s'ajoutaient les excès commis par la guérilla, notamment sous forme de prises d'otage d'éleveurs et de planteurs, de racket généralisé et d'exécution de certains dirigeants politiques locaux. Il était, par ailleurs, évident que la guérilla envisageait d'élargir davantage son emprise sur la région.

Certains, pour venger les pertes subies, n'hésitent pas à recourir, à titre individuel, à des bureaux d'assassins à solde à Turbo, à Medellín ou ailleurs ${ }^{40}$, pour faire éliminer des dirigeants syndicaux, politiques ou civiques, supposés faire partie des réseaux communistes ou maoïstes. Cela n'en reste pas moins une stratégie extrêmement dangereuse qui risque toujours de se retourner contre le commanditaire. Les associations de planteurs (Augura) et d'éleveurs (Fadegan) faisaient bien savoir que la guérilla était en train de s'emparer d'Urabá et exigeaient sans cesse que l'Etat prenne les mesures nécessaires pour défendre les institutions et la propriété privée dans la région. Mais in situ, tandis que la force publique se montrait incapable de contrôler la situation, les planteurs, éleveurs, commerçants etc. se voyaient bien obligés, à titre individuel, de conclure des accords avec l'un ou l'autre des groupes armés - en payant des rançons ou en acceptant de payer une 'protection' - pour pouvoir maintenir leurs affaires dans la région. Dans ce monde, dès le début hétéroclite et atomisé, en résultait une ambiance de défiance qui renforçait encore la difficulté de s'accorder entre planteurs, éleveurs, etc. pour mener des actions sur un plan collectif.

Nous l'avons dit, des actions paramilitaires et para-policières ont toujours existé dans la région à l'initiative de certains secteurs de la société régionale en collaboration avec la force publique et ce, le plus souvent, dans le but de supprimer des formes de délinquance commune ou de banditisme. Or, il s'agit maintenant de faire front à des groupes de guérilla de plusieurs centaines de personnes bien organisées et fortement armées. L'organisation de contre-actions demande désormais non seulement un certain niveau de coordination, mais aussi des ressources financières considérables ${ }^{41}$.

50 Là où les réseaux traditionnels étaient en fin de compte incapables d'agir, les narcotrafiquants, de plus en plus implantés dans la région, proposent des 'solutions'. Ils se chargeront, en effet, de monter une campagne d'éliminations de dirigeants de l'extrême gauche et une vague de massacres contre des bases supposées de la guérilla. Grâce aux moyens de corruption des narcotrafiquants, mais aussi du fait d'intérêts coïncidents, et tout en pouvant compter sur des appuis importants, non seulement parmi les élites régionales, mais aussi parmi la force publique, ils introduisent des groupes paramilitaires dans la région, entre autres à partir de Magdalena Medio, où règne déjà une guerre locale entre réseaux mafieux et guérilla FARC.

51 L'aspect le plus systématique de cette campagne vise les communistes de l'UP, très présents dans la région, et accusés de double jeu en voulant participer à la scène politique légale tout en entretenant des contacts plus qu'ambigus avec les FARC ${ }^{42}$. A Urabá, des dizaines de représentants de l'UP (parmi eux des maires, des conseillers municipaux, des dirigeants syndicaux, des membres de comités syndicaux) sont 
éliminés ${ }^{43}$. Les réseaux maoïstes (dont les dirigeants restent dans la clandestinité) comptent en même temps leurs propres victimes.

En ce qui concerne les massacres collectifs, les épisodes les plus connus à Urabá sont ceux de mars 1988, en période préélectoral, dans les bananeraies Honduras et La Negra, et de Punto Coquitos. Dans le premier cas, des commandos paramilitaires, opérant en pleine nuit, en présence d'un délateur, exécutent, devant les portes de leurs baraques, 20 travailleurs dont les noms figurent sur une liste. Les victimes sont accusés d'être des sympathisants, ou même des guérilleros, de l'EPL temporairement détachés en tant que travailleurs bananiers sur ces bananeraies. Le choix des victimes n'est pas gratuit. En fait, les champs limitrophes à ces deux bananeraies - sur lesquelles le propriétaire absentéiste exerçait au moment des faits un contrôle flou - avaient été transformés en camps d'entraînement par l'EPL. A Punto Coquitos, 26 personnes sont assassinées dans des circonstances confuses, mais apparemment pour cause d'invasion collective, sous la direction de l'EPL, de terrains hautement stratégiques car situés au bord de la mer, au nord de Turbo, et appartenant à un narcotrafiquant. En 1988, quatre autres massacres perpétrés par les paramilitaires ont eu lieu dans la région, faisant 32 victimes supplémentaires.

Le massacre de la Honduras et la Negra, dans la mesure où il vise des travailleurs à l'intérieur d'une plantation, provoque un tel impact sur l'opinion publique nationale et même internationale, qu'une grande enquête judiciaire est pour la première fois menée dans la région. En résultent, en fin de compte, les condamnations (sans conséquence) de certains militaires, installés à l'époque dans la région, du narcotrafiquant Fidel Castaño, tenu pour responsable intellectuel de l'opération. Si Fidel Castaño, condamné par contumace, n'a jamais été arrêté, les paramilitaires ne durent pas moins battre quelque peu en retrait.

La guerre de la réinsertion (1991-1994)

54 En mars 1991, après des négociations de paix extrêmement rapides avec le gouvernement de César Gaviria (1990-1994), l'EPL décide, de manière tout à fait inattendue, d'abandonner les armes ${ }^{44}$. La réinsertion de l'EPL est reçue avec optimisme dans la région. Or, elle finit paradoxalement par provoquer une nouvelle guerre entre les deux factions de l'extrême gauche, mettant fin au pacte (précaire) qui régnait entre elles depuis la fin de la guerre syndicale en $1986^{45}$. Il s'agit, cette fois-ci, d'une guerre locale presque ouverte, les deux protagonistes principaux ne cachant plus leurs objectifs.

55 Cette décision intervient dans une conjoncture nationale favorable, mais avec pour arrière-fond le risque d'une confrontation généralisée, dans la région d'Urabá, entre Fidel Castaño et l'EPL, suite à une provocation de ce dernier sur des territoires du narcotrafiquant, stratégiquement situés sur la rive gauche du golfe d'Urabá. Furieux, Castaño aurait décidé d'entamer une guerre d'extermination contre les réseaux maoïstes, comparable à celle menée contre l'UP et les réseaux communistes en général. Les dirigeants de l'EPL - ayant atteint la quarantaine après vingt années de lutte armée - optent alors pour les conditions généreuses d'amnistie, de grâce et de réinsertion sociale et politique offertes par le gouvernement, non sans avoir la plus grande difficulté à convaincre une partie des commandants et des combattants de leurs Fronts.

56 Ayant abandonné la lutte armée, les ex-dirigeants de l'EPL ne veulent pas pour autant quitter la scène politique. Sous les bannières d'une nouvelle formation politique, Esperanza, Paz y Libertad, les ex-commandants de Fronts ou les dirigeants clandestins 
devenus les nouveaux cadres de Esperanza, prévoient de fonder leur future légitimité politique, notamment dans la région d'Urabá, en mettant à leur profit leurs réseaux d'emprise syndicaux et urbains encore présents. Ils espèrent aussi profiter de la lassitude de la population régionale face à la violence, qui s'était en effet massivement et spontanément manifestée dans les rues d'Urabá, le jour même de l'abandon des armes par l'EPL, en mars $1991^{46}$.

L'adieu aux armes des maoïstes est respecté par les communistes, d'autant plus que les FARC prévoient de pouvoir s'approprier les territoires de l'EPL dans la région. Par contre, l'ambitieux projet politique d'Esperanza relève, pour les communistes, de l'inacceptable, car celui-ci met directement en cause leur contrôle sur les administrations municipales dans la région. L'UP risque en effet de perdre sa majorité dans le conseil municipal et, à la limite, le contrôle des mairies face à une opposition de l'EPL allié avec les plus grandes factions libérales.

L'enjeu de la guerre de réinsertion, qui se déclenche en 1992, est encore une fois essentiellement territorial. D'une part, Esperanza et ses nouveaux alliés qui visent à (re)conquérir un espace politique aux dépends des communistes. D'autre part, les FARC, qui se lancent dans une stratégie de récupération des territoires EPL, notamment les zones rurales et les plantations auparavant sous contrôle des maoïstes, et qui renforcent en même temps leur contrôle politico-militaire sur les quartiers populaires, via leurs milices urbaines.

La région va de nouveau assister à un cycle infernal d'assassinats et de massacres au coup par coup, notamment dans les bananeraies et dans les villes-champignons. Les assassinats sont perpétrés, listes de noms en main, et font souvent 5 à 10 victimes à la fois. Parmi les quelque 500 personnes assassinées dans le cadre de cette guerre, figurent, entre autres, une centaine des 670 ex-combattants de l'EPL ayant abandonné les armes à Urabá (los reinsertados). Les autres victimes sont essentiellement des dirigeants syndicaux, des candidat conseillers municipaux ou des membres de coopératives de l'une ou de l'autre faction, ainsi que des travailleurs bananiers ou des habitants de quartiers populaires sous contrôle de l'une ou de l'autre faction. Certains ex-combattants de l'EPL ayant intégré des groupes de police rurale sont particulièrement visés. Sont aussi éliminés des administrateurs de plantations, des dirigeants libéraux et d'autres, accusés de collaborer avec les reinsertados.

Les éliminations contre Esperanza sont notamment réalisées par les Milicias bolivarianos (les réseaux urbains des FARC) et par le Grupo Caraballo, formé par des excombattants de l'EPL ayant abandonné le processus de paix à l'instigation du dirigeant historique Francisco Caraballo, qui espère à titre individuel récupérer et prolonger l'héritage de l'EPL. Les dirigeants d'Esperanza, avec l'accord tacite des institutions, réorganisent leurs anciennes milices urbaines sous le nom de Comandos populares. Ces derniers vont opérer dans les quartiers populaires et dans les campements des bananeraies pour défendre la population contre les FARC et le Grupo Caraballo. Cela dit, les Comandos Populares passent aussitôt à leur tour aux actions de vengeance contre des bases sociales des FARC.

61 La guerre de la réinsertion prend provisoirement fin le 23 janvier 1994, avec un massacre faisant 35 victimes lors d'une fête à Apartado dans La Chinita, un quartier populaire sous la coupe d'Esperanza. Le massacre, qui prend place en période préélectorale, a été attribué aux milices urbaines des FARC (Milicias bolivarianas) ${ }^{47}$. Dans les semaines qui suivent, plus de 100 membres de l'UP, parmi eux trois des quatre 
maires UP et les principaux dirigeants régionaux, seront arrêtés dans la région, puis inculpés pour complicité et écroués dans les prisons de Medellín et de Bogota ${ }^{48}$.

La deuxième guerre anti-subversive (1995-1997)

62 La région espérait, avec la réinsertion de l'EPL, une diminution de la violence (organisée). Elle assistait par contre à une multiplication des fronts de guérilla ${ }^{49}$ et des groupes armés moins organisés (Comandos populares, le Grupo Caraballo, les Milicias bolivarianas). L'attitude des FARC est ouvertement guerrière ${ }^{50}:$ des attaques sont lancées un peu partout dans la région, contre des biens publics et privés. L'incapacité de la force publique à faire face à cette prolifération de bandes armées privées et l'impunité totale qui règne dans la zone, malgré un renforcement significatif du dispositif judiciaire depuis 1991, est impressionnante. La " solution " à ce " chaos " est apparemment supposée venir des forces sur le terrain même.

C'est dans ce contexte qu'on assiste, à partir 1994, à la réactivation de l'expérience paramilitaire. Des Autodefensas Campesinas de Cordoba y de Urabá apparaissent, de nouveau prises en charge par Fidel Castaño (et son frère Carlos), mais cette fois clairement sollicités par certains secteurs régionaux, décidés à mettre de l'ordre dans la maison ${ }^{51}$. Dans une région lassée de quinze années de guerres locales, ce projet de mise au pas généralisée, qui passe par la grande terreur, semble rencontrer l'assentiment d'une partie plus ou moins importante de la population régionale qui ne demande pas autre chose que d'expulser la guérilla, c'est à dire les FARC, hors la région. Cette deuxième guerre anti-subversive se traduit par une sorte de campagne de nettoyage systématique, qui passe du nord au sud de la région. Est visé tout un chacun qui n'est pas prêt à choisir son camp. Les paramilitaires visitent chaque hameau; ceux qui 'collaborent' avec la guérilla ont deux jours pour partir; ceux qui partent sont bien entendu les premiers à perdre la vie; d'autres, dénoncés, subissent le même sort.

En utilisant la grande terreur, les Autodefensas affirment battre la guérilla avec ses propres armes. Toutefois, ce n'est désormais plus seulement par le nombre de victimes, mais aussi par la cruauté que celle-ci s'impose. On décapite les victimes, on place parfois les têtes sur des bâtons, et on interdit la récupération des corps par les proches. Sont ainsi reprises des logiques de La Violencia, qu'on croyait définitivement remplacées par la simple exécution à balle.

Durant les six premiers mois de 1995, il y a plus de 250 homicides dans la seule municipalité de Necocli (zones rurales incluses). Dans les territoires très contestés, en particulier la zone bananière, la guerre est particulièrement violente, comme en aoûtseptembre 1995, quand une série de cinq massacres fait 80 victimes $^{52}$. Les conseillers municipaux, les dirigeants syndicaux etc. sont particulièrement visés.

67 Au début de 1997, les Autodefensas de Urabá et les communistes s'accordent sur une chose : la région a été nettoyée de toute présence communiste. Tous les dirigeants politiques de l'UP sont obligés de quitter la région. Les quatre fronts des FARC éclatent et doivent se retirer et se replier dans le Cordoba, ou plus loin encore, et sur la frontière colombo-panaméenne. Des exodes de réfugiés ont lieu vers les centres urbains à l'intérieur de la région, mais aussi hors de celle-ci ${ }^{53}$.

Une nouvelle situation est donc apparue. Une paix armée règne dans la région. Les Autodefensas, contrairement à la guérilla, ne se livrent pas à des attaques contre les institutions publiques (postes de police rurale, inspections de Police, Caisses agraires, tracteurs ou camions, autocars), ou à des prises d'otage contre rançon ${ }^{54}$. L'acceptation 
silencieuse de la nouvelle situation exprime le désir des habitants d'en finir avec ces guerres qui durent depuis 15 ans. Or, on est loin d'une situation où la population a repris en main son propre destin : c'est aussi la silence de la terreur.

La force publique (1976-1996)

Le panorama de la violence stratégique et des guerres locales doit être complété par quelques remarques sur le fonctionnement de la force publique durant la période considérée.

70 En gros, la force publique n'a pas été capable de faire face ni à la généralisation de la violence ni à la prolifération des groupes armés privés. A cela plusieurs raisons. D'abord, la complexité géographique de la région et le type même de conflit qui se livrent dans la région rend très difficile la tâche de la force publique. La grande prédisposition à la violence et le port généralisé d'armes par les bandes armées, les sicaires et beaucoup d'autres, rendent les interventions policières extrêmement dangereuses ${ }^{55}$. Les agents de police ont, par ailleurs, été l'objet d'assassinats commandités de toutes parts. Des stations de police ont été l'objet d'attaques par la guérilla. L'armée n'entre plus dans des zones entières de la région. Le risque d'embuscade y est trop élevé face à des groupes de guérilla désormais constitués de plusieurs Fronts et de centaines de combattants bien armés. Mais il y a des problèmes plus structuraux. Tant la police que les militaires souffrent d'absence de stratégie bien définie pour lutter contre la criminalité organisée et la subversion ${ }^{56}$. A cela, s'ajoute une mauvaise coordination et une séparation imprécise des responsabilités entre la police et les militaires ainsi qu'entre les multiples services de renseignement. Il y a aussi, dans une région comme Urabá, un manque d'implantations décentralisées ${ }^{57}$, laissant des zones étendues sans aucune présence policière ou militaire. Enfin, la faiblesse de l'administration judiciaire, d'autant plus inefficace que ses fonctionnaires subissent régulièrement de graves menaces, n'offre pas le soutien nécessaire au bon fonctionnement de la force publique.

71 Dans ce contexte, une fois la situation à la dérive, l'armée et la police ont, elles aussi, joué la carte de leurs intérêts propres et ont, de ce fait, multiplié alliances et arrangements avec les bandes organisées et moins organisées pour pouvoir se maintenir sans trop de conflits ouverts dans la région. Par conséquent, l'armée et la police rencontrent de sérieux problèmes de décomposition interne. Off the record, des entrepreneurs locaux et bien d'autres témoignent des pratiques de corruption et de participation à la délinquance et au trafic de drogue de certains officiers et d'autres membres de la force publique.

72 Plus grave, cette dernière est responsable de bavures, de brutalités à l'égard de la population civile, de tortures et de disparitions à l'égard des (supposés) membres des groupes armés. L'armée a parfois bombardé des zones rurales. Que la force publique opère non seulement de manière confuse et ambivalente, mais se présente en plus sous le visage de la répression, a largement contribué à son discrédit dans la région ${ }^{58}$.

73 La force publique a prêté, tout au long les années quatre-vingt et quatre-vingt-dix, un soutien subtil aux divers groupes paramilitaires ou para-policiers actifs dans la région. Le problème est que l'Etat colombien a historiquement laissé un espace important à des réseaux privés armés pour maintenir l'ordre ${ }^{59}$, notamment dans les régions périphériques comme celle d'Urabá. C'était une politique moins onéreuse et plus respectueuse des libertés d'action des pouvoirs locaux, que la création d'institutions militaires, policières et judiciaires modernes et efficaces. Les Autodefensas de Urabá 
mettent à profit cet héritage quand ils font référence à l'incapacité de l'Etat à garantir la propriété privée et la vie humaine et évoquent une obligation civique de " rétablissement de l'ordre ", de " restauration du droit " et de " protection à la population civile ". Ainsi, à Urabá, comme il y a dix ans dans le Magdalena Medio ${ }^{60}$, le grand combat contre la guérilla n'est pas le fait de la force publique, mais des organisations paramilitaires mises en place par une alliance confuse de narcotrafiquants et d'élites régionales, appuyées par d'autres secteurs de la population. En autorisant les Autodefensas à nettoyer une région si fortement contestée par la guérilla, on permet aussi à des narcotrafiquants comme les frères Castaño de se transformer en acteur politique.

Dans un effort pour récupérer le contrôle de la situation, le gouvernement d'Ernesto Samper (1994-1998) a décidé de mettre en place des polices privées locales, les Convivir. Il s'agit de coopératives de vigilance privée, intégrées par des personnes salariées et armées, dont les agissements sont formellement garantis par les responsables de la coopérative, qui sont souvent des entrepreneurs, et par un service spécial du Ministère de la Défense, qui délivre aussi des permis de fonctionnement. Les Convivir reprennent, sous une forme institutionnellement mieux encadrée, les expériences antérieures des Grupos de Autodefensa et de la Defensa civil. La politique des Convivir semble aussi $s^{\prime}$ inspirer de l'expérience des Rondas Campesinas péruviennes ${ }^{61}$.

Toutefois, dans une situation aussi polarisée et complexe que celle d'Urabáb2 ${ }^{2}$ ce genre d'initiatives peut difficilement avoir le même caractère que celle des Rondas au Pérou. D'abord, à Urabá, la défaite de la guérilla passe par le grand nettoyage entamé par les Autodefensas, c'est à dire par des hommes forts, et non par la force publique en coopération avec les initiatives locales d'autodéfense civile, comme cela a été le cas au Pérou. Ensuite, pour pouvoir se maintenir dans un contexte de luttes territoriales multiples, les quelques Convivir qui existent aujourd'hui à Urabá ${ }^{63}$ doivent disposer de l'accord tacite des Autodefensas, et plus particulièrement des bases locales décentralisées que ces dernières ont déjà imposé dans presque tous les centres urbains de la région, en reprenant le système des milices urbaines de la guérilla (maintenant déplacées). Enfin, plus que les Rondas, les Convivir, encadrés par l'autorité publique mais instrumentalisés par des intérêts privés, risquent toujours de se transformer en une sorte de milice urbaine 'de droite', ce qui ne fait que renforcer l'image d'une guerre civile locale. D'autant plus que la guérilla leur a aussitôt déclaré la guerre, et en a déjà tué différents membres dans d'autres régions.

Il est logique que l'Etat prenne des mesures pour protéger la population, soumise à la terreur. Toutefois, quand les formes institutionnelles de maintien de l'ordre publique sont extrêmement inefficaces, comme c'est le cas à Urabá, il est peu probable que des formes plus informelles, comme celle des convivir, parviennent à s'imposer ${ }^{64}$.

Perte de repères

La violence stratégique accompagne, depuis bientôt vingt ans, l'évolution de la société régionale. Les interactions, collusions et chocs entre les acteurs violents se présentent sous forme d'une succession de guerres locales, où nous avons distingué schématiquement cinq épisodes. Chaque épisode concerne un conflit pour le contrôle territorial autour d'un enjeu particulier. Ces guerres se livrent plantation par plantation, quartier par quartier, mairie par mairie. Elles opposent des protagonistes dont les alliances sont en permanence changeantes. La guerre entre les deux groupes de guérilla est si forte qu'elle resurgit à tout moment. Mais le panorama de la violence 
stratégique est, en même temps, beaucoup trop compliqué pour qu'on puisse résumer la situation à une guerre de guérilla.

78 Les encadrements armés, les assassinats et les massacres se sont routinisés et, avec eux, la peur, la loi de silence et la connivence avec la mort. Avec la redéfinition des acteurs de la violence et leurs stratégies, l'utilisation de la violence a perdu son caractère modéré, bien que chronique, propre aux années soixante et soixante-dix, pour se généraliser et se diffuser dans tous les domaines. Les nouveaux protagonistes armés semblent recourir à la violence et à la terreur sans aucune autre légitimité que celle qu'ils s'attribuent eux-mêmes. La confusion des fins et des moyens est particulièrement claire dans l'utilisation de la terreur. Urabá atteint, à partir du milieu des années quatre-vingt, comme bien d'autres parties de la Colombie, une situation où la force brute est devenue souveraine ${ }^{65}$.

79 La crise s'accompagne, au moins pour ceux qui vivent dans la région, d'une grande difficulté à rendre intelligible, à donner sens à la violence. On assiste, au delà de la fragmentation clientélaire de la scène politique et de la faiblesse traditionnelle de l'Etat, à la paralysie des régulations institutionnelles, tandis que les repères politiques traditionnelles se brouillent. Sur le plan régional, depuis la fin des années quatre-vingt, il n'y a plus de dirigeants politiques capables de s'imposer, de s'afficher en tant que leaders, de proposer un projet et de faire croire à sa faisabilité. Tout se passe comme si la société régionale se construisait désormais exclusivement sur la base de marchandages locaux. La société même préfère les accommodements à une prise de position ferme, car celle-ci risque toujours de se traduire par de nouveaux conflits. On continue certes à gérer les affaires quotidiennes, mais il s'agit de pseudo-jeux institutionnels, qui cachent mal que l'essentiel se passe ailleurs. Les conventions collectives, la remise des armes de l'EPL, les négociations de paix avec la guérilla, l'introduction d'une nouvelle charte : rien ne semble pouvoir faire barrage à la dérive de la situation.

80 L'absence d'hommes politiques sur la scène locale est, d'une part, le résultat des menaces et des assassinats qui ont frappé leurs rangs. Participer à la politique et candidater comme dirigeant local, conseiller municipal ou maire est devenu une affaire risquée, quelque que soit l'affiliation politique. Peu de gens sont prêts à y jouer leur vie. Ainsi, quand l'assassinat se banalise, celui d'un homme politique local ne provoque plus nécessairement une grande consternation ${ }^{66}$.

81 L'absence de dirigeants est, d'autre part, le résultat du manque de crédibilité et de la fragmentation extraordinaire du champ politique, et ce à un niveau plus général ${ }^{67}$. Les élites sociales et politiques, tout en multipliant les références à l'Etat de droit et au besoin de restaurer le monopole de l'Etat sur la violence, se livrent, à titre individuel ou plus collectif, à des arrangements circonstanciels avec les groupes armés privés pour sauvegarder leurs intérêts économiques et politiques dans la région, au lieu de rétablir l'autorité. La corruption des dirigeants politiques, qui est un phénomène traditionnel, notamment en période électorale, s'est radicalisée dans les années quatre-vingt. Des narcotrafiquants installés à Urabá, ont ouvertement financé des campagnes électorales locales durant ces années ${ }^{68}$. Des réseaux mafieux sont ainsi arrivés à imposer leur emprise sur les réseaux politiques traditionnels et agissent en autorités de substitution.

82 Dans une situation où même les violences les plus stratégiques ne se laissent plus ramener à des dimensions politiques ou sociales, et où la terreur s'impose, la difficulté à donner un sens aux événements est en effet bien réelle. Face aux violences 
stratégiques une certaine intelligibilité est certes possible, mais l'excès et les atrocités rendent de plus en plus aléatoires les efforts d'interprétation des faits en termes de relation de cause à effet. Quand des commandos armés agissent, listes en main, pour sélectionner leur victimes dans des autocars, des campements des bananeraies ou lors des fêtes dans des quartiers populaires, et qu'ils les exécutent ensuite au vu du restant de la population, la capacité à donner un sens à ces événements est bien restreinte.

Encadrements armés

Rappelons qu'à Urabá, ni l'EPL ni les FARC n'ont jamais été les instruments armés de mouvements sociaux, c'est à dire des groupes armés qui tirent leur légitimité politique d'un rapport intime avec certains secteurs de la société régionale. Il s'agit à l'origine de guérillas partisanes, c'est à dire d'organisations venues s'implanter dans la région entre la fin des années soixante et le début des années soixante-dix, à la conquête de bases sociales nouvelles, agissant en tant que bras armés de partis politiques d'extrême gauche, qui visaient la conquête du pouvoir par les armes ${ }^{69}$. Le succès manifeste que la guérilla obtient ensuite avec la conquête de certaines ressources matérielles s'articule à un encadrement de la population, qui aurait dû permettre, en même temps, au moins la recréation d'une apparence de légitimité politico-militaire. Mais elle se traduit en réalité par une dépolitisation et une propension croissante à l'intolérance pour maintenir son emprise sur le social.

Ces pratiques vont bien au delà de celles du closed shop classique. Le travailleur embauché sur une bananeraie devient non seulement obligatoirement membre du syndicat, mais il est aussi considéré comme sympathisant, et même candidat potentiel au recrutement par la bande armée qui règne sur le territoire concerné. L'adhésion au syndicat ne peut être discutée sous peine d'être accusé de trahison, d'être un espion ou un délateur (sapo). La subordination des intérêts syndicaux aux intérêts des groupes armés et la transformation des travailleurs en population otage, finit inévitablement par discréditer, parmi les travailleurs, les nouvelles organisations syndicales, malgré l'amélioration des conditions de travail, et les hausses des salaires obtenues. S'il règne à première vue une remarquable unanimité dans ces secteurs (participation massive aux grèves), celle-ci relève alors aussi d'un régime de terreur.

Les formes de protection armée, imposées aux quartiers populaires des villes champignons, relèvent de la même logique. Le contrôle micro-territorial imposé à la population par des milices se veut être une réponse au manque de régulation institutionnelle. Les milices instaurent en effet des sanctions sévères face à la délinquance et n'acceptent pas que d'autres personnes ou groupes utilisent la violence pour leur propre compte. La présence de ces groupes impliquerait donc une certaine 'pacification', notamment une diminution des violences plus anomiques. Les habitants disent approuver le système de correction imposé aux " asociaux " ou aux " désintégrés ". Toutefois, plus que sollicité, cet " ordre " est lui aussi imposé et maintenu par l'instauration de la peur et au travers de la violence arbitraire (exigence de complicité, exécution de récalcitrants) ${ }^{70}$.

La terreur est efficace du point de vue de celui qui l'applique : l'élimination de l'autre ou son déplacement forcé permet d'établir un contrôle effectif sur le territoire contesté. Il s'agit de couper les ponts, d'éliminer les intermédiaires et de faire fuir les relais de l'organisation rivale parmi la population. La banalisation progressive de l'utilisation de la violence s'exprime sur ce plan par l'augmentation des victimes lors des massacres et, plus récemment, par la cruauté infligée aux corps. Les protagonistes 
rivalisent les uns avec les autres pour montrer qui osera aller le plus loin sur le plan de la brutalité. Ce n'est pas un hasard si le nombre moyen de victimes des massacres était de cinq au milieu des années quatre-vingt, lors de la guerre syndicale ; puis est passé à 20 lors des massacre de Honduras y La Negra à la fin des années quatre-vingt; et passe enfin à 35 dans les années quatre-vingt-dix, avec le massacre de La Chinita, lors de la guerre de la réinsertion.

$87 \mathrm{Au}$ delà des cycles de massacres, il y a presque en permanence une terreur plus quotidienne : l'assassinat ponctuel d'une institutrice, d'un professeur, d'un dirigeant d'association de base, d'un prêtre, d'un dirigeant indigène, d'un dirigeant syndical. Cette terreur quotidienne, constitue le véritable toile de fond de ces guerres locales. Les massacres n'en sont que le côté le plus spectaculaire, bien qu'en même temps le plus révélateur ${ }^{71}$.

Liens circulaires

On estime qu'à Urabá il y a eu, entre 1984 et 1996, quelque 12.000 morts violentes. Dans le département d'Antioquia en général, on attribue pour cette période $25 \%$ des homicides à la violence 'organisée' et $75 \%$ des homicides à la violence désorganisée (violence juvénile, tueurs à gage, délinquance commune, règlements de comptes, décomposition de bandes) ${ }^{72}$. Ces chiffres sont à lire eu regard du poids des chiffres provenant de Medellín, où la violence a un caractère très désorganisé. A Urabá, la violence stratégique est plutôt responsable de 50 à $75 \%$ des homicides, ceci selon les épisodes de guerres locales.

Ce genre d'estimation n'en reste pas moins aléatoire. D'abord, puisque l'impunité quasi-totale qui règne dans la région rend toute attribution de responsabilités provisoire. Ensuite, du fait que, dans une situation de violence généralisée, comme nous la rencontrons à Urabá, la violence stratégique et la violence désorganisée entretiennent des liens circulaires qui brouillent les registres et rendent la distinction même entre l'un et l'autre peu pertinente. Nos dernières remarques concernent précisément la manière dont la violence organisée entretient des rapports circulaires avec des formes de violence plus désorganisées, déstructurées ou anomiques.

La violence juvénile

90 A Urabá, comme dans le reste de la Colombie, se sont des gens souvent très jeunes qui intègrent les bandes armées. Comme se sont souvent des jeunes qui se livrent aux violences les plus désorganisées, ils en sont aussi les premières victimes.

91 Comme l'affirme un ex-commandant de la guérilla EPL : "Pour fonder un groupe armé dans une région comme Urabá, on n'a que besoin d'armes et d'argent, car des jeunes, il $\mathrm{y}$ en a toujours"73. Des jeunes auraient en effet toujours rejoints, de manière relativement spontanée, les rangs des diverses bandes armées qui opèrent dans la région, et cela sans trop se préoccuper de la spécificité " idéologico-politique " de l'un ou l'autre groupe. A Urabá, pour recruter leurs membres, guérilla, paramilitaires et autres se tournent essentiellement vers des secteurs sociaux faiblement intégrés, notamment celui des colons, des petits paysans, des habitants des communes rurales ou des quartiers populaires des villes-champignons (et beaucoup moins celui des travailleurs bananiers $)^{74}$. Ils trouvent là des jeunes politiquement et socialement disponibles, à la recherche d'une identité, avec de faibles espoirs de progrès dans la vie, pour qui les groupes armés peuvent représenter un canal de mobilité sociale, où ils peuvent en plus investir un certain esprit d'aventure et des idées utopiques souvent très naïves ${ }^{75}$. 

le temps. Dans les années soixante-dix, la guérilla recrute ses combattants de manière relativement sélective, et à la condition que ceux-ci disposent d'une certaine formation et d'une certaine expérience politico-idéologique. Dans les années quatre-vingt, l'augmentation des effectifs et l'audace croissante des groupes armés se traduisent par un recrutement plus aléatoire, car plus massif. Il s'agit maintenant d'avantage de recruter des guerriers que des gens politiquement motivés. Le recrutement, tant pour la guérilla que pour des groupes paramilitaires et autres, devient en fin de compte très instrumental. Des jeunes se font désormais recrutés pour des motivations essentiellement pragmatiques : obtenir de $l^{1} \operatorname{argent}^{76}$, fuir le chômage ou le service militaire ${ }^{77}$, bénéficier d'avantages personnels les plus divers. Ces motifs n'excluent pas le recrutement à base de pressions, de menaces ou de chantage sur la famille. Certains se font recruter après avoir reçu des accusations de collaboration, par exemple pour avoir rencontrer un proche impliqué dans une bande rivale. Il y a finalement ceux qui adhèrent par soif de vengeance, ou par simple goût du combat.

En ce qui concerne l'entrée des jeunes dans le monde de la violence plus désorganisée, dont le premier théâtre est celui des villes-champignons ${ }^{78}$, le même argument s'applique. Ceux déjà habitués à vivre de petits boulots dans le secteur informel ou d'activités semi-légales, côtoyant depuis des années les phénomènes de bandes et de milices, passent plus facilement à la délinquance que les jeunes mieux insérés. De leur point de vue, les nouveaux 'boulots' n'apparaissent pas radicalement différents du rebusque quotidien, à ceci prêt qu'ils garantissent immédiatement des profits importants. Le monde de ces jeunes a moins pour toile de fond la pauvreté, qu'un certain manque de normes, de valeurs et de rôles bien définis, dans le double contexte d'une société colombienne en modernisation rapide et d'une société frontalière nouvellement construite, marquée par une certaine désorganisation sociale.

Il n'empêche que l'explosion de la violence juvénile, avant de proliférer de façon relativement autonome ${ }^{79}$, est d'abord le fait d'une réponse à des opportunités concrètes offertes dans le nouveau marché du crime et de la violence organisée. Une explication de la violence juvénile et de la participation des jeunes aux bandes armées exclusivement en termes de phénomènes de désorganisation sociale risque en effet de se transformer en euphémisme, faisant l'économie d'une analyse des responsabilités plus organisées.

La prolifération des sicaires et des bandes

commandants des Fronts de guérilla, les capos du narcotrafic et les chefs des bandes paramilitaires restent en coulisses où ils gèrent les affaires et définissent les coups à porter. Le sale boulot est délégué vers le bas, vers les milices urbaines, les bandes de sicaires, les matones, chargés d'exécuter des 'contrats', de réaliser des prises d'otages, de brûler des autocars, des camions, des installations des bananeraies ou d'autres biens appartenant à ceux qui refusent de s'acquitter du racket. Cette délégation relève, bien entendu, d'une compartimentation commandée par des impératifs de sécurité, mais aussi d'un souci des chefs à ne pas être personnellement impliqués dans les basses besognes et donc de garder les mains (plus) propres.

96 A l'ombre des acteurs organisés, il y a donc une prolifération d'acteurs violents plus désorganisés. Se crée tout un bas monde de jeunes tueurs à gage, d'assassins, d'hommes à tout faire, des bureaux de sicarios relativement autonomes apparaissent. A Turbo, on signale, en 1993, l'existence d'un bureau de sicaires, mené par une femme,

Cultures \& Conflits, 24-25 | hiver 1996- printemps 1997 
communément connue comme Doña $\mathrm{X}$, sans aucune affiliation directe à un des acteurs organisés. Il y des milices de la guérilla, qui, profitant de leur relative autonomie, se transforment en partie en bandes de quartiers.

Autour de ces acteurs plus désorganisés se créent, à leur tour, des conflits pour le contrôle du marché de la délinquance commune, du racket, de contrats d'assassinats etc. Naissent alors des micro-guerres locales sui generis, plus désorganisées et anomiques que les guerres déjà décrites. Des tueries ont lieu dans des bars, sur des terrains de football, à des coins de rue ou à l'école.

Ce panorama des violences plus désorganisées n'est pas un simple effet pervers de la violence stratégique. Que ce bas monde soit disponible convient aux acteurs violents plus organisés, qui aiment à dire, en cas d'excès, qu'ils ont beaucoup de mal à contrôler leurs milices en dérive.

Une sous-culture de la violence

99 A Urabá, comme dans toute guerre, la majorité des gens ne cherche qu'à rester dans l'ombre, à ne pas être mêlés aux groupes armés, à ne pas prendre de risques. La majorité des gens, même parmi ceux qui habitent dans les quartiers les plus touchés, ne participent directement ni à la guérilla, ni aux réseaux du narcotrafic, ni aux groupes paramilitaires, ni aux milices urbaines ou aux autres formes de bandes ayant recours à la violence. Cela vaut aussi pour les jeunes.

100 Cela dit, tous s'inscrivent dans ce qu'on peut appeler, avec précaution, une sous-culture de la violence. Sous-culture sur un plan général, dans la mesure où avec la généralisation de la violence, les gens finissent par avoir recours à ce mode d'interprétation historique qui veut qu'en Colombie le recours à des logiques de violence soit permanent, relève de la tradition, et soit en fin de compte inévitable. Devenus fatalistes et en partie apathiques, les habitants locaux arrivent à parler de 'la violence' comme d'" un autre choléra ", " un autre sida ", une autre épidémie devenue entre-temps la première cause de décès dans la région ${ }^{80}$. Le recours à cette métaphore illustre bien la réapparition, sous condition de la violence généralisée, de l'idée d'une l'histoire nationale colombienne dominée par la violence en tant qu'une force incontrôlable et anonyme ${ }^{81}$. Peut-être que le recours à un imaginaire de la violence en terme de force incontrôlable rend à la limite plus supportable l'inévitable nécessité de s'adapter dans la vie quotidienne à la situation.

101 Sous-culture sur un plan plus concret, dans la mesure où à Urabá chaque personne, sans exception, a un ou plusieurs enfants, amis, proches ou voisins faisant partie des groupes ou bandes armées. Tous ont aussi des proches ayant été victimes d'assassinats. Beaucoup ont été confrontés, directement ou par le biais de récits de proches, aux cruautés, aux brutalités ou aux massacres. Par ailleurs, dans la mesure où les guerres locales ont obligé les gens à quitter les zones rurales et les campements des bananeraies, très touchés par la terreur, les quartiers nouveaux des villes-champignons se peuplent aussi en partie de gens traumatisés par la violence.

102 C'est cette cohabitation quotidienne avec la violence qui fait qu'on peut raisonner, pour les jeunes, en termes d'une génération de la violence, et plus généralement, après 15 années de guerres locales, en termes de l'apparition d'une sous-culture de la violence. Cette cohabitation risque d'apparaitre, au moins de l'extérieur, comme une sorte d'acceptation ou de complicité.

Complicités et profits 
Quiconque habite dans la région est bien obligé de s'adapter d'une manière ou d'une autre à la situation. C'est le cas des éleveurs, payant la vacuna pour se protéger contre le vol de bétail. C'est le cas des planteurs, payant l'impôt révolutionnaire pour ne pas voir incendier des installations ou être menacés de prise en otage. C'est le cas des transporteurs, qui payent aussi l'impôt révolutionnaire, pour que la guérilla ne brûle pas leurs camions.

Mais l'adaptation à la situation prend aussi des modalités plus ambiguës. D'autres intérêts y trouvent en effet leur compte. C'est ce qu'on peut appeler le monde gris des complicités qui sont bien entendu, elles aussi, de toutes sortes et de tous ordres. Ainsi, certains administrateurs des plantations ou de domaines d'élevage, responsables de la gestion des impôts révolutionnaires, profitent de leur rôle d'intermédiaire pour la transformer en affaire lucrative et, du coup, dangereuse. Certains y auraient laissé la vie. Il y a aussi, comme à l'époque de la Violencia, des gens qui profitent de l'insécurité régnante pour acquérir des terrains ruraux ou urbains à bas prix ${ }^{82}$. Certains planteurs plus importants semblent ainsi avoir profité de la crise des plus petits au moment des grèves et de la guerre syndicale, en rachetant les bananeraies en difficultés financières à des prix avantageux. Des spéculateurs urbains n'ont cessé de négocier, durant les années quatre-vingt et quatre-vingt-dix, des terres autour du trajet de la Panamericana, alors situé en plein territoire FARC.

Le phénomène le plus préoccupant est bien entendu le recours de particuliers aux réseaux de la violence, notamment à des tueurs à gage, pour régler des comptes personnels ou plus prosaïques.

Il y a enfin, plus indirectement, l'effet pervers de la migration. Les villes de Monteria et de Medellín accueillent, depuis le milieu des années quatre-vingt, des milliers de déplacés en provenance d'Urabá, réfugiés ou rescapés de l'un ou l'autre massacre. Mais, en même temps, des migrants nouveaux n'ont jamais cessé d'arriver à la recherche d'un avenir meilleur. Avec ces nouveaux arrivants, même si la violence fait chaque année 1.000 morts, les places sont aussitôt reprises par des gens qui connaissent peu ou pas la région et qui préfèrent ne pas en savoir plus. La mémoire de la violence, dans la région même, parait ainsi de courte durée, et on retrouve les récits et les bribes de l'histoire de la région plus facilement hors la région que dans la région même.

7 Si ces logiques ne sont pas à la base de la crise actuelle, elles n'en ont pas moins facilité l'installation durable d'une conjoncture de violence généralisée. Avec ce genre de régulations informelles, parfois des plus surréalistes, l'économie régionale, au-delà de certaines périodes particulièrement difficiles, n'a jamais cessé de prospérer.

L'impunité

Les cercles vicieux de la violence généralisée sont aussi entretenus par une impunité quasi totale. La quasi totalité des homicides commis dans la région depuis le fin des années soixante-dix sont restés impunis. Des recherches judiciaires n'ont jamais été entamées dans la majorité des cas. Moins que 3\% des homicides sont élucidés. A cela essentiellement deux raisons. Premièrement, l'inefficacité notoire de l'administration de la justice, en particulier de l'instruction criminelle. A part la violence proprement organisée, des dizaines de (jeunes) tueurs à gage circulent en toute liberté dans la région. Deuxièmement, l'impunité pré-judiciaire, dans la mesure où les gens se méfient de la justice et ont terriblement peur de représailles s'ils portent plaintes ou témoignent. Depuis des années, à Urabá, la très grande majorité des dossiers d'instruction criminelle concernant des cas d'homicide ne sont constitués que d'une 
seule feuille : celle de la nécropsie, aucun témoignage n'ayant pu être obtenu. Les gens respectent en effet strictement certaines règles de survie : ne jamais se mêler des affaires des autres et toujours faire comme si on n'avait ni rien vu ni rien entendu. Il faut savoir s'adapter aux règles informelles d'une société de sourds, de muets et d'aveugles, comme un habitant de la région l'expliquait de manière tout à fait percutante.

109 L'impunité n'en est pas moins une stratégie. Tardivement créé, faiblement organisé et fortement clientélisé, l'appareil judiciaire connaît traditionnellement un manque chronique d'indépendance. Les élites politiques et sociales usent et abusent de la justice pour la contourner à leur profit. La Colombie, en fin de compte, a toujours été un pays où règnent les hommes et non les lois. Mais elle l'est aujourd'hui plus que jamais. Car l'impunité est aussi voulue et entretenue par les protagonistes armés, qui opèrent par définition dans l'illégalité et obtiennent leur impunité par un recours à la terreur. A Urabá, des juges, des avocats, des inspecteurs de police, etc. sont constamment victimes de menaces et d'exécutions commanditées par les réseaux mafieux, mais aussi par la guérilla, qui exige sans cesse le respect des droits de l'Homme ainsi que l'installation d'observateurs internationaux à Urabá, mais n'a jamais fait du renforcement de la justice une priorité. Personne n'exige en effet, au delà de la présence d'observateurs internationaux, l'installation d'une commission de la vérité sur les 12.000 homicides des 15 dernières années à Urabá, pour que la lumière soit faite sur les responsabilités collectives et individuelles de ces crimes.

110 Nous le voyons, l'impunité même est ainsi une illustration des liens circulaires entre violence organisée, dérégulation institutionnelle et désorganisation sociale. Une désorganisation sociale, qui résulte en partie des modes particuliers de construction de la région, est à la base de certaines formes de violence. La désorganisation sociale, dérégulation sociale et institutionnelle, est une conséquence de la violence (organisée).

Conclusion

Bien que, dans une grande mesure, la culture de l'élite et la culture populaire en Basse Meuse aient constitué des modèles l'une pour l'autre et l'une de l'autre, il serait pourtant certainement exagéré de dire qu'elles se sont développées dans une relation mutuellement constitutive. Mais il y avait une bonne part de " reflet " - imitation, mime, parodie - de la part des groupes subalternes, tandis que la violence des châtiments reflétait quelque chose de l'échelle, de l'ampleur, de la forme et du sens des crimes, réels et imaginaires. La violence massive que les deux groupes s'infligeaient réciproquement (sans précédent dans l'histoire de la République hollandaise et étrangement négligée dans l'historiographie officielle hollandaise) et son réalisme explicitement magique ne peuvent être compris que si l'on se réfère aux caractéristiques les plus marquantes de ces territoires : leur fragmentation politique, leur localisation périphérique, et leur structure seigneuriale qui faisaient se côtoyer splendeur aristocratique et misère plébéienne. Ces conditions fournissaient à l'évidence un espace considérable aux groupes dissidents 42 , mais provoquaient la répression et définissaient ses modalités. Confrontés à des formes massives et prolongées de subversion, les membres de la classe dirigeante restauraient leur domination par le biais du théâtre de la loi, étouffant par la même occasion les voix contestataires. Le tribunal (salle d'audience), le lieu de détention, la rue et le lieu de l'exécution fournissaient le cadre de cette affirmation d'hégémonie culturelle, permettant, tout en introduisant un élément dramatique, de mettre l'accent sur la distinction entre le corps 
intègre d'une part, et le corps violé, déshonoré, pourrissant d'autre part - distinction qui, par un jeu métonymique (pars pro toto), permettait une restauration magique de l'ordre.

\section{NOTES}

1.Cet article a été réalisé dans le cadre d'une recherche (1993-95) financée par la Fondation pour la Recherche Tropicale (WOTRO) de l'Organisation Néerlandaise de Recherche Scientifique (NWO).

2.A l'époque de La Violencia, la Colombie avait quelque 15 millions d'habitants, contre 30 millions en 1985 et 35 millions en 1995.

3.Dans ce qui suit, si nous parlons de violence, nous faisons toujours référence à une violence meurtrière.

4.Voir notamment les articles suivants de Daniel Pécaut, " Réflexions sur la violence en Colombie ", in Françoise Héritier (dir.), De la violence, Paris, Odile Jacob, 1996, p. 225-271 ; " Présent, passé, futur de la violence ", in Jean-Michel Blanquer et Christian Gross, La Colombie à l'aube du troisième millénaire, IHEAL, Paris, 1996 ; " Violence et politique en Colombie ", in François Dubet, Michel Wieviorka (dir.), Penser le Sujet. Autour d'Alain Touraine, Paris, Fayard, 1995 ; " Violence et politique: quatre éléments de réflexion à propos de la Colombie ", in Cultures \& Conflits, $n^{\circ} 13-14$, printemps-été 1994 ; " Crise, Guerre et Paix ", in Problèmes d'Amérique Latine, 1987.

5.Au sujet de la terreur, voir plus en particulier l'article de Daniel Pécaut dans le présent numéro de Cultures \& Conflits.

6.Daniel Pécaut, " Crise, Guerre et Paix ", op. cit.

7.Daniel Pécaut, " Réflexions sur la violence en Colombie ", op. cit., p. 226.

8.Cf. Jenny Pearce, Colombia, Inside de Labyrinth, Latin American Bureau, 1990 ; Diana Duque Gomez, Una guerra irregular entre dos ideologias. Colombia 1982-1990, Bogota, 1991.

9.Cf. James B. Rule, Theories of civil violence, University of California Press, 1988.

10.Daniel Pécaut, " Réflexions sur la violence en Colombie ", op. cit.

11.Daniel Pécaut, " Présent, passé, futur de la violence ", op. cit.

12.Nous laissons donc de côté d'autres dimensions d'une situation de violence généralisée, en particulier la dégradation de la dimension politique et la construction d'un imaginaire de la violence.

13.Le grand Medellín, avec quelque 2.5 millions d'habitants, soit $8 \%$ de la population nationale, totalise environ 8.000 homicides par an, soit le quart du total national. La région d'Urabá, avec 350.000 habitants, soit $1 \%$ de la population nationale, totalise environ 1.000 homicides par an, soit 3,3\% du total national.

14.Dans le sens de José Matos Mar, Desborde popular y crisis del Estado. El nuevo rostro del Peru en la década del 80, Lima, IEP, 1984, qui décrit l'irruption de l'économie informelle, l'apparition d'énormes bidonvilles populaires ainsi que la crise des emprises politiques, institutionnels et religieux traditionnels suite à l'explosion démographique de Lima pour cause de migrations internes. 
15.Dans le sens de W. I. Thomas et Florian Znaniecki, The polish peasant in Europe and America, Boston, Gorham Press, 1920, qui insiste sur la perturbation de l'univers normatif des migrants polonais avant et après leur migration vers les Etats-Unis. 16.Pour l'histoire de la région d'Urabá, voir James J. Parsons, Antioquia's Corridor to the Sea. An historical geography of the settlement of Urabá, Berkeley, 1967. Pour des analyses de la situation actuelle, voir notamment Fernando Botero, Urabá.

Colonizacion, violencia y crisis del Estado, Medellín, Universidad de Antioquia, 1990 ; Clara Inez Garcia, Urabá, Region, Actores y conflicto. 1960-1990. Medellín, INER, 1996 ; Maria Teresa Uribe, Urabá : region o territorio? Un analisis en el contexto de la politica, la historia y la etnicidad, Medellín, INER, 1992.

17.Le secteur réalise quelque 300 millions dollars US en chiffre d'affaires (1995), ce qui représente environ $15 \%$ du marché mondial de la banane. Les bananeraies couvrent une superficie totale de 25.000 hectares (1995), entourées de larges zones d'agriculture paysanne (100.000 hectares) et d'élevage (350.000 hectares).

18.D'autres villes champignons de moindre poids démographique mais avec une fonction sub-régionale importante, se créent dans les zones proprement paysannes de la région. Il s'agit notamment de San Pedro de Urabá et de Bajira.

19.On distingue notamment, parmi les migrants, des morenos de la côte Atlantique, des negros de la côte Pacifique, des chilapos de Cordoba et des antioquenos de l'intérieur du département.

20.Le régime du Front National (1958-1974), où libéraux et conservateurs se partageaient tous les postes publics et installèrent une alternance obligée au niveau présidentiel, fut conçu comme un régime de transition pour mettre fin à la Violencia et préparer le passage à une démocratie ouverte.

21.Pour le passage de la violence chronique vers la violence généralisée en Colombie, voir Daniel Pécaut, " Réflexions sur la violence en Colombie, op.cit.; " Présent, passé, futur de la violence ", op.cit.

22.Les FARC ont été fondés en 1966 dans le sud du pays, autour de certains groupes de paysans encadrés par le PCC (fondé dans les années 30). Dans les années 60, le PCC et l'état-major des FARC estiment que les conditions à Urabá ne sont pas encore favorables à l'implantation d'un front de guérilla. Certains combattants y sont par contre recrutés et y retourneront plus tard pour aider à la constitution du 5ème front. Sur les origines des FARC, voir Eduardo Pizarro Leongomez, Las FARC. De la autodefensa a la combinacion de todas las formas de lucha, Universidad Nacional, Bogota, 1991. Pour l'EPL, voir Alvaro Villaraga et Nelson R. Plazas, Para reconstruir los suenos. Una historia del EPL, Bogota, Fundacion Progresar, 1994.

23.Dans les années 60 et 70, le PCC, en profitant de la fragmentation des libéraux, participait régulièrement à des coalitions majoritaires à l'intérieur des conseils municipaux, ce qui permettait d'obtenir des cuotas burocraticos, notamment des postes publics à Turbo, Apartado et Chigorodo.

24.En 1982, les FARC, pour marquer leur passage à une action plus offensive, ajoutent à leur sigle les lettres EP (Ejercito Popular).

25.L'existence depuis 1987 d'une Coordinadora Guerrillera Simon Bolivar (CGSB), qui réunit la majorité des groupes de guérilla colombiens, cache mal la fragmentation dont souffre la guérilla en tant qu'acteur. Par ailleurs, chacun de ces groupes (M-19, ELN, EPL, FARC, ADO, etc.) se constitue de plusieurs fronts qui opèrent de façon relativement autonome, en fonction de conditions et d'objectifs locaux. Nombre de ces fronts manquent, par ailleurs, de cohésion interne et souffrent d'infiltrations et de désertions. 
La paranoïa atteint un tel niveau que les chefs du groupe Ricardo Franco, un noyau dissident des FARC, ont éliminé, en 1987, plus de 200 combattants accusés de trahison. 26.Parmi ces réseaux actifs à Urabá on retrouve déjà Pablo Escobar, qui aurait commencé sa carrière criminelle en 1969, par des affaires de contrebande entre Medellín et le port de Turbo. En 1975, le futur chef du cartel de Medellín a déjà fait de la cocaïne son produit de contrebande le plus important, il continue à utiliser le Golfe d'Urabá pour transporter la mercancia vers les Etats-Unis et ailleurs, introduit entre autre dans des bateaux de bananes. A partir 1977, Escobar installe des laboratoires de cocaïne à Urabá et au Magdalena Medio. A Medellín, le début de l'essor de l'économie de la cocaïne s'accompagne, à partir 1973, d'une augmentation de 10\% par an des cas d'homicides. Cf. Luis Canon, El Patron. Vida y Muerte de Pablo Escobar, Bogota, Planeta, 1994, p. 51, 59, 77.

27.Cf. Simon Strong, Whitewash. Pablo Escobar and the cocaïne wars, MacMillan, London, 1995, et Luis Canon, op.cit. p. 214.

28.José Ocampo, collaborateur de la première heure de Escobar, construit au début des années 80 à Nécocli, une impressionnante ferme équipée d'un héliport, d'une piste d'atterrissage et d'une antenne parabolique, visible de la route toute proche. Pablo Escobar aurait lui-même disposé d'une maison " de vacances " sur la rive gauche du Golfe, à quelques kilomètres de la frontière Colombo-panaméenne. Fidel Castaño - chef militaire d'Escobar pour cette région avant de devenir, à partir 1991, un de ses ennemis les plus redoutés - a construit durant les années 80 un véritable empire dans le nordouest du département de Cordoba, tout en achetant des grandes domaines à Urabá. Des narcotrafiquants moins connus, mais localement très important, comme la famille Velez et Hugo Lopez, construisent aux années 80 des micro-empires locaux à Urabá. 29.Au delà des enjeux locaux, les purges entre FARC et EPL des années 70 s'inscrivent dans le contexte de conflits idéologiques entre communistes et maoïstes un peu partout dans le monde. Mais, dans leur intolérance extrême, ils reprennent à leur compte la tradition politique nationale d'une division du corps social en deux sous-cultures partisanes (conservateurs et libéraux) marquée par des haines pré-politiques et des confrontations violentes répétées.

30.Le passage de Currulao aurait déjà été l'objet de conflits violents en 1975-76, c'est à dire au moment où le trafic de cocaïne commence à s'imposer dans la région.

31.Cf. Alvin W.Gouldner, Wildcat strikes, New York, Harper Torchbooks, 1965 (1ed. 1954).

32.La majorité des salariés étaient embauchés sur la base de contrats individuels (65\%) ou de pactes laboraux (25\%). Un pacte laboral se distingue d'une convention collective en ce qu'il s'agit d'un contrat de travail signé entre travailleurs et patrons sans l'intervention d'un syndicat. Le champ syndical colombien est en principe dominé par des syndicats d'entreprise.

33.Dans une certaine mesure, une fois la centralisation et l'institutionnalisation des négociations collectives acceptées, le patronat profitait de la discipline imposée par les syndicats de l'extrême gauche, mettant fin aux insécurités liées aux grèves sauvages ou à la concurrence salariale au sein des différentes plantations.

34.Les dirigeants politiques maoïstes, en charge de la région d'Urabá dans les années quatre-vingt, avaient, en partie, participé personnellement à l'infiltration et à la radicalisation des syndicats de textile à Medellín au cours des années soixante-dix, avant de se déplacer vers Urabá, pour y reprendre leurs expériences avec le syndicalisme bananier encore peu organisé. 
35.Les nouvelles conventions instaurent le versement automatique et obligatoire de $2 \%$ du salaire des 12.000 travailleurs des bananeraies en tant que cotisation syndicale. (Sur le plan national, la cotisation syndicale est en général de $1 \%$ du salaire). A cela s'ajoutent des prestations patronales exceptionnelles pour les syndicats.

36.L'Union Patriotica (UP) est fondée en 1985 par les FARC (bras armé du PCC), après la signature d'un cessez-le-feu avec le gouvernement de Belisario Betancur (1982-86). L'UP se présente comme une plate-forme d'unité de toute la gauche, mais ses candidats et membres ne sont pas moins quasi exclusivement des militants du PCC.

37.Les maires sont traditionnellement nommés par les gouverneurs selon des principes de clientèle. En 1986, les gouverneurs sont obligés de nommer des maires sur la base des résultats des élections municipales. A Apartado, les libéraux sont majoritaires, mais fortement divisés. L'UP obtient alors la mairie d'Apartado (et de Mutata). Depuis 1988, les maires sont élus par suffrage direct. A Urabá, l'UP obtient alors, en plein période de guerre anti-subversive, les mairies d'Apartado, de Turbo, de Chigorodo, de Murindo et de Mutata. Sur la combinaison des stratégies politiques, syndicales et armées chez la guérilla colombien, voir Carlos E. Agudelo, De la lutte armée à la combinaison des formes de combat. La Colombie, 1985-95, mémoire de l'IHEAL, 1996.

38.Les maoïstes boycottent les élections de 1986 et jouent à fond la carte syndicale. Les communistes, voulant obtenir la mairie à travers des coalitions habiles avec certaines fractions libérales, étaient obligés de se montrer, malgré tout, plus modérés sur le plan syndical.

39. Complexification dans le sens d'un changement qualitatif suite à la transformation d'un conflit bi-parti à un conflit multi-parti. Voir sur ce point la sociologie de conflit de Georg Simmel (dyades et triades), repris par T. Caplow, Deux contre un, Paris, PUF, 1971 (1968).

40.Des assassins à solde ont toujours existé, mais ils se créent aux années 80 des bureaux plus ou moins autonomes qui acceptent de réaliser des contrats, service utilisé non seulement par les narcotrafiquants, mais aussi par bien d'autres secteurs. Voir notamment Carlos Miguel Ortiz, " El sicariato en Medellín : entre la violencia politica y el crimen organizado ", in Analisis Politica, ${ }^{\circ} 14,1991$, p. 60-74

41.De là qu'on a parfois cité comme étant impliqués dans la coordination des actions paramilitaires des maisons de commercialisation de la banane ou certains parmi les plus grands éleveurs ou planteurs, qui possèdent jusqu'à vingt plantations, mais rarement des planteurs à titre individuel.

42.La guérilla concevait les pourparlers de paix avec le gouvernement de Belisario Betancur comme un manœuvre tactique. Ils en profitaient pour réorganiser leurs fronts et pour renforcer leurs structures légales (UP, syndicats, associations de quartiers etc.)

43.Sur le plan national ont été assassinés, depuis 1985, quelques 2.000 membres de l'UP, sans qu'on puisse attribuer la totalité des victimes aux actions des groupes paramilitaires. Des victimes dues à des exécutions internes, aux conflits régionaux avec d'autres groupes armés, des vengeances plus personnelles, s'y juxtaposent.

44.L'abandon des armes de l'EPL intervient suite à l'abandon des armes en 1990 par le M-19 et d'autres groupes de guérilla de moindre importance, dans un contexte politique marqué notamment par l'élection d'une assemblée constituante et l'élaboration d'une nouvelle constitution (1991). Les FARC, avec quelque 7.000 combattants distribués en 70 fronts, et l'ELN avec quelque 3.000 combattants distribués en 30 fronts, sont désormais les groupes de guérilla colombiens les plus importants. 
45.Après 1986, FARC et EPL continuent à s'affronter à Urabá, par milices urbaines interposées, pour étendre leurs zones d'influence, en particulier dans les quartiers populaires des villes champignons, un champ de bataille ici quelque peu négligé. En 1987, FARC et EPL ont notamment menés un assaut commun contre le village de Saiza. Cette opération, que la guérilla entendait comme le début d'un soulèvement régional général, s'était d'ailleurs soldée par un bain de sang généralisé suite à la résistance des groupes civils d'autodéfense armée.

46.La lassitude de la violence et la délégitimation progressive de la lutte armée, était aussi ressentie par l'UP, dont l'élimination systématique des militants n'arrivait plus à mobiliser à Urabá.

47.Le massacre de la Chinita peut être compris comme une action de vengeance en réponse au massacre de 20 " sympathisants " communistes dans les bananeraies La Ceja et Cativos, le 9 décembre 1994, attribué aux Comandos populares.

48.Les élections de 1994 mettent un point définitif au règne de l'UP à Urabá : elle perd 4 de ses 5 mairies qu'elle dirigeait depuis 1988. A Apartado, à l'issue d'un consensus politique, un candidat unique, la charismatique Gloria Cuartas, sans affiliation politique bien que d'origine conservateur, est installée pour trois ans à la mairie. Laurée le 8 mars 1997 à l'UNESCO à Paris, à l'occasion de la journée internationale de la femme, Gloria Cuartas a entre autre dirigé une Comision Verificadora, dont l'Informe final (octobre 1995, 58 p.) souligne que tous les protagonistes de la violence organisée à Urabá, les forces publiques inclues, sont responsables de graves infractions au droit international humanitaire.

49.Les FARC passent à Urabá, au début des années quatre-vingt-dix, de 2 à 4 fronts : le 5ème opérant dans la zone bananière, le 34ème vers Mutata, le 57ème sur la frontière avec le Choco et le 58ème vers Arboletes. L'ELN, a droit à une espace à Urabá en tant que membre de la CGSB.

50.Fin 1994, quand l'armée annonce des grandes opérations à Urabá, les FARC déclarent accepter le défi. Communiqué du Bloque José Maria Cordoba, El Tiempo, janvier 1995 51.Avec la mort de Pablo Escobar, en décembre 1993, termine la guerre contre le Cartel de Medellín que Fidel Castaño menait, en parallèle avec la force publique, avec d'autres Persécutes de Pablo Escobar (PEPES), une alliance de circonstance où participaient aussi des membres du Cartel de Cali. Les Autodefensas de Urabá reprennent à leur compte une partie des effectifs des PEPES ainsi que son discours auto-légitimateur.

52.Le 12 août 1995, six policiers et militaires accusés de collaboration avec les Autodefensas de Urabá sont assassinés dans un bar à Apartado, par un commando armé des FARC. Quelques heures plus tard, dans un quartier populaire de Chigorodo, ce massacre est vengé par l'exécution de 18 personnes, supposées être des sympathisants des FARC. Le 18 août, un commando des FARC exécute 5 personnes dans les environs d'Apartado. Le 29 août, 19 personnes (sympathisants EPL?) sont exécutes (par les FARC?) sur une plantation de banane dans les environs de Carepa. Le 25 septembre, un commando armé des FARC arrête aux environs d'Apartado un bus et exécute, liste de noms en main, 24 personnes aux yeux des autres passagers. Le 13 et le 14 mai 1995 les Autodefensas de Urabá assassinent à Turbo un total de 21 " sympathisants des FARC ", comme représaille pour l'assassinat de deux de leurs membres.

53.Cf. CINEP, " Urabá : el mayor exito de los ultimos anos. Analisis de la situacion de derechos humanos y desplazamiento en la région de Urabá ", Santafé de Bogota, junio 1995. 
54.Les Autodefensas de Urabá se sont par contre livrés à des prise d'otage à l'encontre de proches parents des commandants des fronts de guérilla ou des membres de l'Etat Major des FARC, pour obliger les FARC à libérer des gens pris en otage pour des raisons financières (parmi eux des éleveurs etc. de la région de Urabá), des membres de la force armée ou des Autodefensas faits prisonniers.

55.En 1993, 280 policiers furent assassinés en Colombie.

56.L'amélioration des services de renseignement, notamment depuis la guerre contre le narcotrafic et grâce à une assistance américaine, a permis l'arrestation ou l'élimination de certains capos des réseaux mafieux et de quelques commandants de la guérilla. 57.Il n'y a jamais eu plus de 3.000 soldats effectivement installés à Urabá, région grande comme la moitié des Pays-Bas mais très accidentée et largement dépourvue de routes. La guérilla rassemblait en même temps environ 800 hommes lourdement armés. 58. Certains efforts d'épuration et de réorganisation de la force publique (notamment de la police) ont été entrepris depuis le début des années quatre-vingt-dix, dans le cadre de la nouvelle Constitution (1991), et suite à de graves accusations nationales et internationales concernant les violation des droits de l'Homme et d'autres brutalités commises sur la population civile. Le paradoxe est que, depuis que la force publique a été obligée de mettre fin à des collaborations trop ouvertes avec des groupes paramilitaires, ces derniers ont récupéré à Urabá un pouvoir sans précédent. 59.Le droit à l'organisation de Grupos de Autodefensa, instauré par le loi 48 en 1968, fut abrogé en 1989, comme conséquence des graves actes de violence perpétrés par divers groupes se réclamant de cette loi, mais fonctionnant hors encadrement institutionnel formel, souvent encadrés par des réseaux mafieux. La Defensa civil, instaurée par une loi de 1964, était une forme d'autodéfense civile légale mise sur pied dans certains hameaux ruraux à l'encontre des phénomènes de banditisme. Mal armée et peu soutenue par la force publique, qui déserte dans les années quatre-vingt, les zones rurales pour cause d'insécurité et manque de moyens, la Defensa civil a été une expérience marginale, par ailleurs peu analysée. La guérilla en a exécuté parfois les membres, tenus pour des collaborateurs (sapos) de la force publique, et cela souvent face aux autres habitants, obligés d'assister au " tribunal populaire ". Rappelons toutefois qu'en 1987, à Saiza (Urabá ), la Defensa civil locale avait fait front, avec succès, à la guérilla (voir ci-dessus).

60.Sur le Magdalena Medio, cf. Carlos Medina Gallego, Autodefensas, paramilitares y narcotrafico en Colombia, Bogota, Ed. Documentos Periodisticos, 1990.

61.Cf. Carlos Ivan Degregori (ed), Las Rondas Campesinas y la derrota de Sendero Luminosa, Lima, IEP, 1996.

62.Le conflit dans les zones les plus violentes a essentiellement été, au Pérou, un conflit entre forces armées et guérilla, tandis qu'en Colombie, dans une région comme Urabá, la guerre entre guérilla et force publique semble relever de contradictions secondaires à côté de celles qui opposent paramilitaires et guérilla et groupes de guérilla entre eux. La situation colombienne se distingue par la multiplicité des acteurs armés.

63.En 1996 existait à Urabá une poignée de Convivir, mis notamment en place par le secteur bananier.

64.Cf. Allan V. Horwitz, New-York, The logic of social control, 1990.

65.Daniel Pécaut, Cronica de dos décadas de politica colombiana. 1968-1988, Siglo XXI, Bogota, 1988, p. 30-31. 
66.L'assassinat d'un dirigeant politique ne relève par ailleurs pas nécessairement de motivations politiques. Il peut être motivé par des éléments plus prosaïques (dettes, affaires ou passions).

67.Les gens sont en effet convaincus qu'un pacte d'unité nationale entre libéraux et conservateurs, comme celui célébré en 1958 pour mettre fin à la Violencia, est peu vraisemblable dans les conditions actuelles d'une fragmentation totale du champ politique (bien que libéraux et conservateurs dominent toujours le champ politique) et n'apportera surtout pas de solution concrète, étant donnée la complexité de la crise. 68.Pour donner un exemple : à Necocli, en 1986, José Ocampo soutenait publiquement la campagne électorale municipale libérale.

69.Il ne s'agit donc pas d'anti-mouvements sociaux qui ont progressivement abandonné un projet social ou politique par l'inflexion des fins et des moyens et qui livrent désormais des actions de terreur contre l'Etat, mais de groupes armés privés en concurrence pour la conquête de territoires, de ressources et de populations en faisant usage de la violence et du terreur. Ce qui n'empêche pas ces protagonistes d'avoir un discours politique pour légitimer leurs actions.

70.Voir sur ce point les réflexions de Daniel Pécaut dans ce numéro de Cultures \& Conflits.

71.Pour une analyse des massacres en terme anthropologique, Cf. Maria Victoria Uriba, T. Vasquez, Enterrar y Callar, 1995, Comité Permanent pour les Droit Humains, Bogota, 1995.

72.Cf. C. Echandia et R. Escobeda, Violencia y desarollo en el municipio colombiano, 1987-1993, Bogota, rapport de la Présidence de la République, 1994, cité par Daniel Pécaut, op. cit., 1997.

73.Entretien avec un ex-commandant de l'EPL, Medellín, 1993.

74.Parmi les 2.150 combattants de l'EPL qui abandonnent en 1991 les armes (dont 670 dans la région d'Urabá), $21 \%$ était analphabète, $56 \%$ avait une suivi éducation primaire, $15 \%$ une éducation secondaire et $8 \%$ était professionnel ou technicien.

75.Pour une caractérisation des bases sociales de l'EPL, Cf. Maria Victoria Uriba, Ni canto de gloria, ni canto funebre. El regreso del EPL a la vida civil, Cinep, Bogota, 1993. Voir pour des analyses comparables sur Sendero Luminoso, Carlos Ivan Degregori, El surgimiento de Sendero Luminoso. Ayacucho 1969-1979, Lima, IEP, 1990 ; " Cosechando tempestades ", in Degregori (ed.), op. cit., p. 190-193.

76.Les Autodefensas campesinas de Cordoba y de Urabá auraient mieux payé leurs combattants que la guérilla et auraient même distribués des motos parmi des centaines de jeunes.

77.Les paramilitaires, tout comme la guérilla, prennent soin d'acheter pour leurs membres la libreta militar, document crucial qui prouve qu'on a effectué son service militaire.

78.Apartado et Turbo disposent, au début des années quatre-vingt-dix, chacun de quelque 50.000 habitants en zone urbaine.

79.Au sujet de la violence juvénile comme sous-culture, notamment à Medellín, $\mathrm{Cf}$. Alonso Salazar, No nacimos pa'semilla, La cultura de las bandas juveniles de Medellín, Cinep, Bogota, 1990 (Il en existe une traduction en français).

80.Urabá a été affecté, au début des années quatre-vingt-dix, par l'épidémie du choléra et connaît un très haut indice de maladies vénériennes, $\mathrm{y}$ inclus le sida.

81.Cf. Daniel Pécaut dans ce numéro. 
82.Voir sur ce point, pour la période de La Violencia, les excellentes analyses de Carlos Miguel Ortiz, La Violence en Colombie, racines historiques et sociales, Paris,

L'Harmattan, 1990 (1ed. Esp. 1985).

\section{RÉSUMÉS}

Since the end of the 1980s, the Colombian region of Urabà and more generally the whole country has been faced with a crisis of violence one could qualify of generalised violence. The author shows that this crisis was firstly not percieved as a group of deviant behaviours but as an carrer opportunity in a new sector of the informal economy. Thus, many strategies of violence are short term strategies in an immediate poximity. The action does not unfurl in a regional context but rather in very small local contexts. The stakes of the action are the control of legal or illegal ressources. But the complexity of the situation and the resulting social disorganisation make it more difficult for people to give a meaning to violence. Violence is therefore experienced as a situation of terror.

\section{INDEX}

Mots-clés : Stratégie, violence, crises politiques

Index géographique : Amérique du Sud, Colombie

\section{AUTEUR \\ GÉRARD MARTIN}

Doctorant à l'EHESS. 